\title{
Dynamics of Polynesian Subsistence: Insights from Archaeofauna and Stable Isotope Studies, Aitutaki, Southern Cook Islands ${ }^{1}$
}

\author{
Melinda S. Allen, ${ }^{2,3}$ and Facqueline A. Craig ${ }^{2}$
}

\begin{abstract}
Human colonists of Remote Oceania readily took advantage of the naive virgin fauna encountered on previously uninhabited islands, a bounty that was quickly depleted. Subsequent developments in Polynesian subsistence economies were more subtle, varied, and complex. These features are illustrated in a comparison of two quite different subsistence archives from the postcolonization period: archaeofaunal assemblages and stable isotope $\left(\delta^{13} \mathrm{C}\right.$ and $\left.\delta^{15} \mathrm{~N}\right)$ records of humans, pigs, and dogs from the same archaeological contexts. The samples come from four stratified sites, with a total of 22 distinct occupational strata that represent a 600 -year period on the small $\left(18.4 \mathrm{~km}^{2}\right)$ almost-atoll of Aitutaki in the southern Cook Islands. Benefits and challenges of integrating these quite different records are considered in the context of specific findings, with implications for subsistence studies elsewhere. In particular, differences in formation processes, taxonomic resolution, and contrasting spatial and temporal scales represented by each record are highlighted. A complex, multiscalar picture of subsistence change emerges, showing variability within and across the three species and the two subsistence archives. Findings support prior interpretations that established (not colonial) settlements are represented by the currently known Aitutaki archaeological record. Within the relatively stable and largely anthropogenic food web, humans occupy a central position throughout the sequence. Through time, a reduction in fishing and decreased consumption of marine carnivores is indicated; these changes are likely to be an outcome of both repeated storm events and considerable shoreline disruption in the fourteenth century A.D., and cultural decisions about the relative costs and benefits of various fishing activities vis-à-vis other subsistence needs. An apparent reduction in variability of pig diets in late prehistory could reflect interspecific competition between pigs and their human managers, although small sample sizes constrain interpretations. Overall, use of two quite different subsistence archives provides a more robust, but also more complex, view of subsistence change across individuals and communities on Aitutaki.
\end{abstract}

\footnotetext{
${ }^{1}$ Funding provided by Wenner Gren Foundation for Anthropological Research to M.S.A. and R.C. Dunnell (1989) and J.A.C., M.S.A. and J. Littleton (2001), National Science Foundation (M.S.A.), University of Auckland Research Committee (M.S.A.), and the Royal Society of New Zealand (Littleton, M.S.A., and J.A.C.). Manuscript accepted 30 January 2009.

2 Department of Anthropology, University of Auckland, Private Bag 92019, Auckland, New Zealand.

${ }^{3}$ Corresponding author (e-mail: ms.allen@auckland .ac.nz).
}

Pacific Science (2009), vol. 63, no. 4:477-506

(C) 2009 by University of Hawai'i Press

All rights reserved
As human colonists spread across Remote Oceania, a process that began around 2,800 years ago, they encountered rich, virgin faunas with few natural defenses. Land birds, seabirds, nesting turtles, reptiles, bats, and other native vertebrates were easy targets, exploited to the extent that they now serve as critical markers of colonizing sites (e.g., Anderson 1994, Steadman 2006). In the main, reliance on these fauna was unsustainable, and intensive harvesting rapidly led to numerous extinctions (e.g., Steadman et al. 1994, Anderson 1997, Nagaoka 2002a, Steadman 2006). Other prey were "depressed" (sensu Charnov et al. 1976), slowly reduced 
to economically unproductive and demographically unstable levels (e.g., Butler 2001, Allen 2002, McAlister 2002, Nagaoka 2002b, Morrison and Hunt 2007). Over the long run, these processes led to marked subsistence change and in some cases even contributed to island abandonment (e.g., Anderson 2001, Weisler 1995).

In the centuries that followed, Polynesians developed more stable subsistence economies, typically built around plant and animal domestics that were complemented by more resilient native resources (e.g., Kirch 1973, Kirch and Yen 1982, Rolett 1998, Walter 1998). In contrast to the relatively uniform boom-bust experiences of early island colonists, change in the postcolonization period was more subtle, complex, and geographically varied. Different combinations of domesticates were emphasized, depending on local conditions, and in some cases (notably small islands) animal competitors were extirpated (Bay-Peterson 1984, Kirch 2000, Giovas 2006). Although marine foods were widely utilized, preferred habitats and species changed over time, in response to human impacts, environmental perturbations, and cultural dictates (e.g., Spennemann 1987, Leach et al. 1999, Allen 2002, Barber 2003, Masse et al. 2006, Morrison and Cochrane 2008). Although less dramatic than early subsistence transformations, accurately discerning the character and causes of postcolonization change is important because that is the period when unique island cultures developed and in the process shaped the Polynesian biota and landscapes that are today the object of ecological study and conservation.

Our understanding of prehistoric Polynesian subsistence economies owes much to the study of archaeofaunal remains, culturally deposited bones, and shell found in archaeological sites. Problematically, however, even large samples from multiple contexts (a rarity) will not necessarily provide accurate records of past human activities (see also Fitzpatrick and Kataoka 2005). This is because archaeofaunal remains can be affected by cultural disposal patterns, taphonomic processes (e.g., weathering), the cultural context of deposition (i.e., site function), as well as archaeo- logical recovery techniques (e.g., sampling procedures and screen size). These potential sources of bias are not unique to archaeofauna assemblages, being a concern for many kinds of archaeological data; evaluating their potential impact requires other independent paleoecological records, most usefully including those that have different formation histories.

In this paper, we compare a 600 -year-long archaeofaunal record from the small island of Aitutaki in the southern Cook Islands with newly available stable isotope analyses (carbon and nitrogen) of human, pig, and dog samples from the same sites and occupation layers. The aims of this comparison are twofold. The first is methodological, specifically a consideration of how these two diverse subsistence archives complement, extend, and/or challenge one another. In comparing the two records, the potentials and biases of different formation processes, information content, taxonomic resolution, and spatiotemporal perspectives are evaluated, with implications for dietary and subsistence studies elsewhere.

The second aim of this paper is to use Aitutaki's archaeological record as a case study of Polynesian subsistence in the postcolonization period and evaluate ideas about the nature and causes of change and stability. The value of Aitutaki as a case study lies in its rich archaeofaunal record, built from several localities and multiple occupation layers, along with a newly available isotopic record. As a small island, it also offers insights into both the challenges of island living and the development of sustainable solutions. Small islands often register the dynamics of human-environmental interactions more quickly and more clearly than larger landmasses, where the possibilities of alternative resources and migration are greater. Through the unique combination of archaeofaunal and isotopic records, we explore both large-scale subsistence change and individual records of diet within and across species. The Aitutaki records point to a complex set of interactions between humans, animal domesticates, and the environment over the ca. 600-year period of study, and the possibility of multiple causal agents contributing to subsistence change. 


\section{Background}

Aitutaki, the northernmost of the eight main islands that compose the southern Cook group, is located ca. $200 \mathrm{~km}$ from its nearest neighbor (Figure 1). This "almost-atoll" consists of a peripheral reef and reef flat (typically 0.5 to $1.0 \mathrm{~km}$ wide) that enclose a relatively shallow (11 m maximum), roughly triangular, $50 \mathrm{~km}^{2}$ lagoon (Stoddart and Gibbs 1975). The $16 \mathrm{~km}^{2}$ mainland is the weathered remnant of the original volcanic core and rises to a maximum of $86 \mathrm{~m}$. Another $2.4 \mathrm{~km}^{2}$ is partitioned amongst 12 coral detritus islets that run along the eastern lagoon periphery, a sand cay in the southwest corner of the lagoon, and two small volcanic islets within the lagoon: Moturakau and Rapota. The three main marine habitats are the lagoon, where coral heads and ridges are interspersed with fine sand; the reef flats (Figure 2); and the outer reef "slope," a near-vertical face of considerable depth.

Although the timing of human settlement in the southern Cook Islands is much debated (i.e., Anderson 1994, Kirch and Ellison 1994), direct archaeological evidence is no earlier than the eleventh century A.D. (see Allen and Wallace 2007). The Ureia (AIT-10) site in particular was once considered an early occupation locality, with an initial age estimate of ca. A.D. 1000 (Bellwood 1978, Allen 1994). Further analyses, however, along with 14 new radiocarbon determinations, now place settlement here in the period A.D. 1225-1430 ( $1 \sigma$ age range [Table 1]) (Allen and Wallace 2007). Basal occupation strata at other Aitutaki sites also fall within or after the thirteenth century A.D. (Table 2). Only Tangatatau Rockshelter on Mangaia Island (Kirch et al. 1995), with a small number of eleventh- to twelfth-century determinations, suggests earlier settlement but, given that they are on unidentified wood charcoal, their accuracy cannot be fully assessed. Importantly, other independent evidence that might definitively identify any of these localities as early settlement sites is lacking or equivocal.

Aitutaki's archaeosedimentary records provide key insights into the environmental history of the island. Geoarchaeological study (Allen 1998) suggests formation of the mod- ern beach ridge coincident with late Holocene sea level fall, a phenomenon dated to ca. A.D. $800-1200$ in the nearby Tuamotu Islands (Pirazzoli and Montaggioni 1986, Dickinson 2003). By the fourteenth century A.D., the western coast of Aitutaki was sufficiently stable to allow human settlement, although not without frequent incursions by storm surf (Figure 3). Excavations at several localities along the west coast exhibit aggrading sequences, the product of alternating cultural occupations and storm events (Allen 1998). The recent redating program outlined earlier now places two, and possibly three, major storm events in the fourteenth century A.D. A pene-contemporaneous event is registered on Moturakau Islet (Zone E), suggesting that at least one tropical cyclone impacted both the exposed west coast and the inner lagoon. Similarly, Walter (1998) recorded a substantial storm deposit on Ma'uke Island to the south in the fourteenth century.

Today the southern Cook Islands regularly experience tropical cyclones, particularly in combination with El Niño weather patterns (Salinger and Lefale 2005, de Scally 2008). On average the group is buffeted by $1.8 \mathrm{cy}-$ clones per season, and since 1891 events large enough to cause major human damage have occurred every 8.8 years (de Scally 2008). After major tropical cyclones (i.e., hurricanes) coral reefs can take several decades to recover (Stoddart 1985). Specifically on Aitutaki, residents noted that fish distributions and migration patterns were disrupted and ciguatera fish poisoning increased after Cyclone Sally in 1987 (Adams et al. 1996). El Niño conditions have damaged Aitutaki's reef in other ways as well. Pauley (1994 in Adams et al. 1996) found that the unusually warm sea temperatures that accompanied El Niño conditions in 1991, 1992, and 1994 resulted in coral bleaching and mortality both within the lagoon and along the fore-reef, reaching up to $90 \%$ for some species; a subsequent outbreak of crown-of-thorns (Acanthaster planci) led to further reef deterioration. These modern impacts are relevant to our consideration of the fourteenth-century storm events noted earlier and the patterns of subsistence change we discuss herein. 


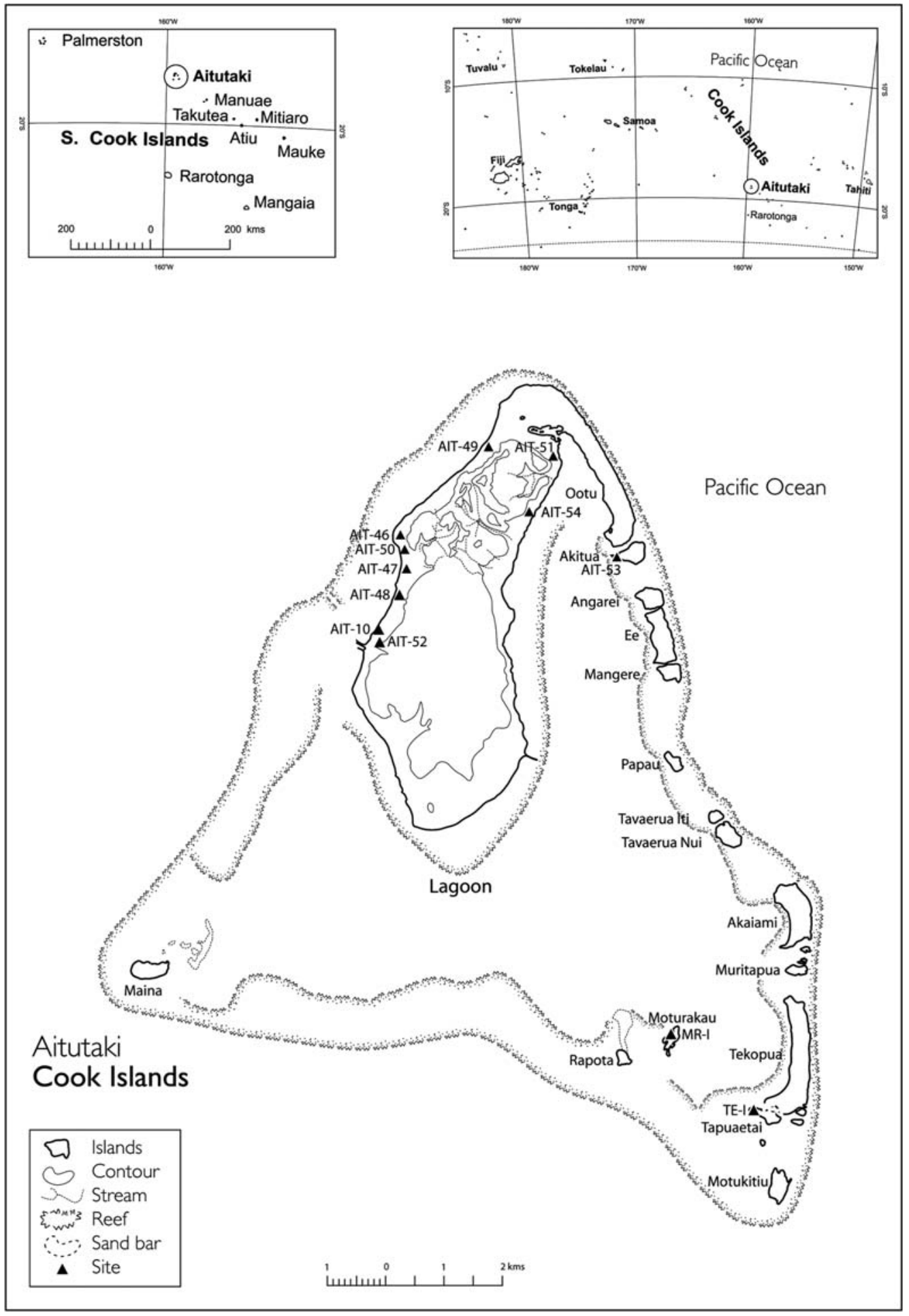

FIgURE 1. Aitutaki, southern Cook Islands, and archaeological sites referred to in text. 


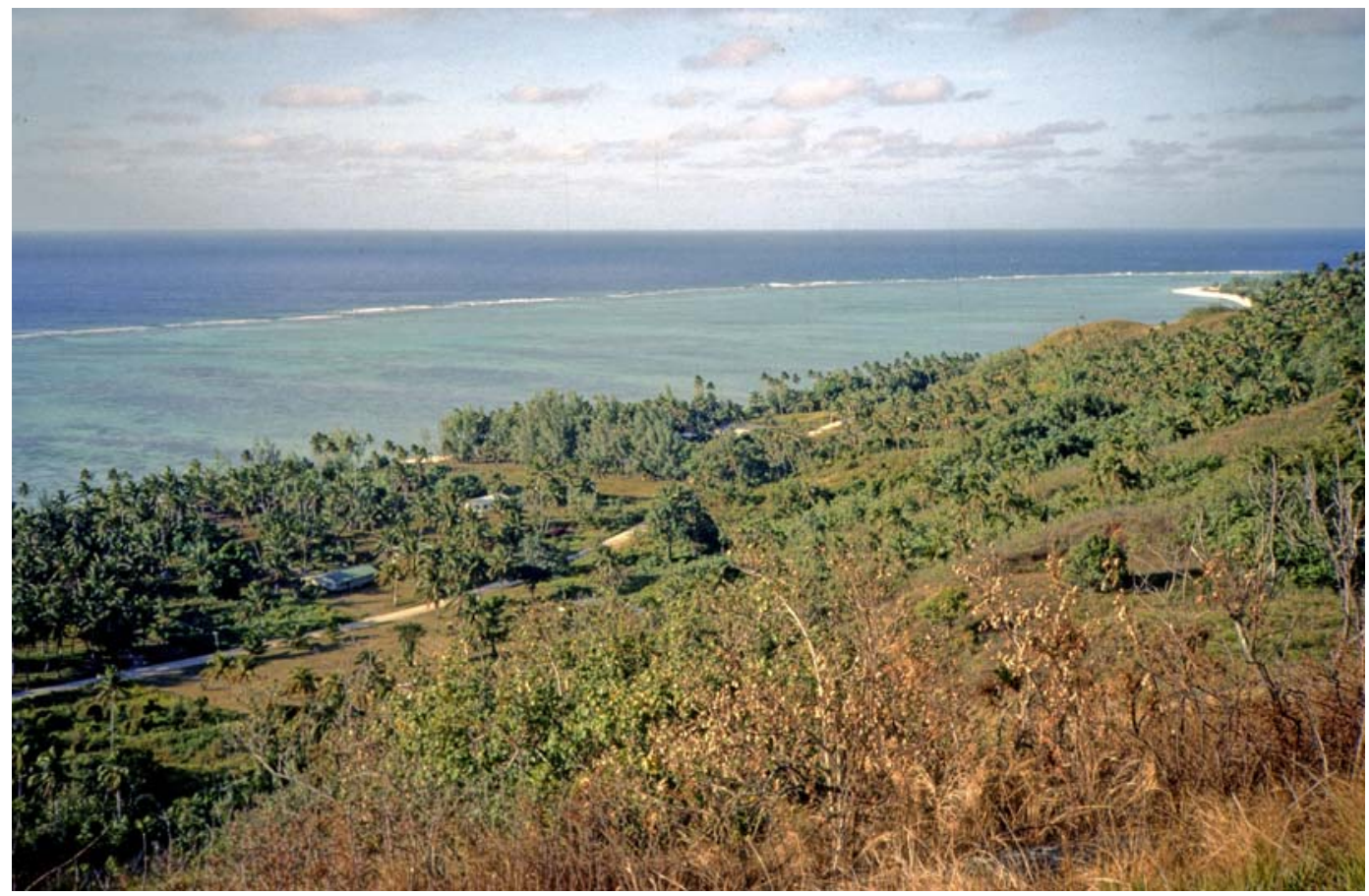

FIGURE 2. Looking down on the narrow coastal flat and fringing reef of Aitutaki's west coast.

The samples used in this study derive from three stratified sites on the west coast of the mainland and a rockshelter on Moturakau Islet $\left(0.04 \mathrm{~km}^{2}\right)$ in the lagoon (Figure 1 , Tables 3 and 4). The Ureia site was excavated first by Bellwood (1978), who uncovered but did not formally excavate burials in the upper cultural zone of the site (Zone C). Allen (1992) subsequently excavated additional areas, as well as other sites on the island. All of the materials reported on herein come from Allen's excavations, with the exception of a small number of human bone samples excavated by Bellwood at Ureia that were used in the stable isotope study.

Cultural strata at the three mainland sites of Ureia (AIT-10), Hosea (AIT-50), and Aretai (AIT-49) represent relatively permanent residential areas and are broadly functional equivalents, based on analysis of the local stratigraphy, features, and artifacts (Allen 1992). Moturakau Rockshelter (MR-1), in contrast, contains a series of short-term occu- pations associated with intensive fishing activities and adze manufacture. Given the small size of this islet, and the lack of a permanent water source, in all likelihood the rockshelter occupants permanently resided on the Aitutaki mainland.

\section{MATERIALS AND METHODS}

\section{Faunal Analysis}

A total of 19,892 faunal specimens (excluding unidentified fish) was recovered from the four sites, with the bulk of the remains coming from Moturakau Rockshelter (Table 5). Although procedures varied somewhat between sites (see Allen [1992] for details), in all cases the excavated sediments were processed with $6.4 \mathrm{~mm}(1 / 4 \mathrm{inch})$ or finer mesh screens and the fauna extracted in a field laboratory. The faunal materials were identified using published sources and several reference collections, including specimens from Bishop Museum and three personal collec- 
TABLE 1

Details for Radiocarbon Samples from Ureia Site (AIT-10)

\begin{tabular}{|c|c|c|c|c|c|c|}
\hline $\begin{array}{l}\text { Laboratory } \\
\text { No. }{ }^{a}\end{array}$ & Provenience & $\begin{array}{l}\text { Conventional } \\
{ }^{14} \text { C Age B.P. }\end{array}$ & $\begin{array}{l}\text { Type of } \\
\text { Analysis }\end{array}$ & $\begin{array}{l}\text { cal. B.P. } 1 \sigma \\
\text { range }^{b}\end{array}$ & $\begin{array}{l}\text { cal. A.D. } 1 \sigma \\
\text { range }^{b}\end{array}$ & Material $^{c}$ \\
\hline \multicolumn{7}{|c|}{ Prior determinations } \\
\hline Beta-25251 & Zone C & $200 \pm 50$ & Standard & Post-290 & Post-1660 & Wood charcoal \\
\hline Beta-25248 & Zone E & $720 \pm 60$ & Standard & $680-560$ & $1270-1390$ & $\begin{array}{l}\text { Wood charcoal and } \\
\text { Cocos endocarp }\end{array}$ \\
\hline Beta-25249 & Zone E & $760 \pm 60$ & Standard & $739-560$ & $1220-1390$ & $\begin{array}{l}\text { Wood charcoal and } \\
\text { Aleurites } \text { endocarp }\end{array}$ \\
\hline Beta-27439 & $\begin{array}{l}\text { Zone J } \\
\quad(\text { level 13) }\end{array}$ & $790 \pm 70$ & Standard & $740-570$ & $1210-1380$ & $\begin{array}{l}\text { Wood charcoal and } \\
\text { Cocos endocarp }\end{array}$ \\
\hline \multicolumn{7}{|c|}{ New determinations } \\
\hline WK-18401 & $\begin{array}{l}\text { Zone G } \\
\quad \text { (level 7) }\end{array}$ & $692 \pm 29$ & AMS & $655-560$ & $1295-1390$ & $\begin{array}{l}\text { Monocot }(\text { Cocos? }) \text { and } \\
\text { broadleaf }\end{array}$ \\
\hline WK-18402 & $\begin{array}{l}\text { Zone G } \\
\quad \text { (feature 4) }\end{array}$ & $703 \pm 29$ & AMS & $660-565$ & $1290-1385$ & Thespesia populnea \\
\hline WK-19119 & $\begin{array}{l}\text { Zone G } \\
\quad(\text { level 7) }\end{array}$ & $717 \pm 52$ & Standard & $670-560$ & $1280-1390$ & $\begin{array}{l}\text { Cocos endocarp, } \\
\text { Hibiscus, Cordia, } \\
\text { Artocarpus, Pemphis }\end{array}$ \\
\hline OZI-982 & $\begin{array}{l}\text { Zone G } \\
\quad(\text { level 9) }\end{array}$ & $560 \pm 45$ & AMS & $555-510$ & $1395-1440$ & Cordia subcordata \\
\hline WK-18408 & $\begin{array}{l}\text { Zone G } \\
\quad \text { (level 9) }\end{array}$ & $756 \pm 31$ & AMS & $680-570$ & $1270-1380$ & Cordia subcordata \\
\hline OZI-983 & $\begin{array}{l}\text { Zone G } \\
\quad \text { (feature 3) }\end{array}$ & $760 \pm 70$ & AMS & $730-560$ & $1220-1390$ & $\begin{array}{l}\text { Guettarda, Cordia, } \\
\text { Tournefortia }\end{array}$ \\
\hline WK-18404 & $\begin{array}{l}\text { Zone J } \\
\quad \text { (level 12) }\end{array}$ & $642 \pm 36$ & AMS & $635-550$ & $1315-1400$ & Cocos endocarp \\
\hline WK-18410 & $\begin{array}{l}\text { Zone J } \\
\quad \text { (feature 6) }\end{array}$ & $727 \pm 32$ & AMS & $670-570$ & $1280-1380$ & $\begin{array}{l}\text { Terminalia, Cocos } \\
\text { endocarp }\end{array}$ \\
\hline WK-18403 & $\begin{array}{l}\text { Zone J } \\
\quad \text { (level 12) }\end{array}$ & $799 \pm 29$ & AMS & $725-665$ & $1225-1285$ & Broadleaf taxon \\
\hline WK-18406 & $\begin{array}{l}\text { Zone J } \\
\quad \text { (level 14) }\end{array}$ & $663 \pm 29$ & AMS & $640-555$ & $1310-1395$ & Cocos endocarp \\
\hline WK-18405 & $\begin{array}{l}\text { Zone J } \\
\quad \text { (feature 4) }\end{array}$ & $670 \pm 29$ & AMS & $650-555$ & $1300-1395$ & Cocos endocarp \\
\hline OZI-981 & $\begin{array}{l}\text { Zone J } \\
\quad(\text { level 15) }\end{array}$ & $590 \pm 45$ & AMS & $630-520$ & $1320-1430$ & Cocos endocarp \\
\hline WK-18407 & $\begin{array}{l}\text { Zone J } \\
\quad \text { (level 15) }\end{array}$ & $644 \pm 29$ & AMS & $635-550$ & $1315-1400$ & Cocos endocarp \\
\hline WK-18409 & $\begin{array}{l}\text { Zone J } \\
\quad(\text { level 16) }\end{array}$ & $692 \pm 30$ & AMS & $655-560$ & $1295-1390$ & Cocos endocarp \\
\hline
\end{tabular}

Note: From Allen and Wallace (2007); samples of uncertain provenience, gross outliers, those that lack ${ }^{13} \mathrm{C} /{ }^{12} \mathrm{C}$ ratios, and uncalibrated marine shell are excluded (see original publication for details).

${ }^{a}$ Determinations prefaced with WK- were prepared at the Waikato Radiocarbon Laboratory and the Accelerator Mass Spectrometry (AMS) determinations undertaken by Rafter Radiocarbon Laboratory, GNS Science. Sample prefaces indicate the following laboratories: OZI- provided by Australian Nuclear Science and Technology Organisation and Beta- by Beta Analytic Inc. (USA).

${ }^{b}$ Samples were calibrated with OxCal 3.10 (Bronk Ramsey 1995, 2001) using the Southern Hemisphere calibration curve (McCormac et al. 2004).

${ }^{c}$ All material is wood charcoal unless otherwise indicated.

tions (itemized in Allen [1992: appendix F.1]). Most of the vertebrate materials were quantified as number-of-identified-specimens (NISP) to avoid the problems of aggregation associated with minimum-number-of- individual calculations (Grayson 1984, Allen 1992). In considering the relative abundance of fish, however, the NISP counts are based solely on the five paired jaw elements (dentary, articular, quadrate, premaxilla, and max- 
TABLE 2

Details for Radiocarbon Samples from Other Aitutaki Sites

\begin{tabular}{|c|c|c|c|c|}
\hline Laboratory No. & Provenience & $\begin{array}{l}\text { Conventional } \\
{ }^{14} \text { C Age B.P. }\end{array}$ & $\begin{array}{l}\text { cal. B.P. } \\
1 \sigma \text { range }\end{array}$ & $\begin{array}{l}\text { cal. A.D. } \\
1 \sigma \text { range }\end{array}$ \\
\hline \multicolumn{5}{|l|}{ Aretai (AIT-49) } \\
\hline Beta-40762 & Zone E & $310 \pm 60$ & $450-150$ & $1500-1800$ \\
\hline Beta-34330 & Zone G & $400 \pm 50$ & $500-320$ & $1450-1630$ \\
\hline \multicolumn{5}{|l|}{ Hosea (AIT-50) } \\
\hline Beta-41062 & Zone E & $320 \pm 70$ & $479-150$ & $1490-1670$ \\
\hline Beta-31604 & Zone G & $620 \pm 80$ & $730-340$ & $1300-1430$ \\
\hline \multicolumn{5}{|c|}{ Moturakau (MR-1) } \\
\hline Beta-44371 & Zone A & $230 \pm 50$ & $310-140$ & $1640-1810$ \\
\hline Beta-41572 & Zone B & $50 \pm 60$ & 250-Modern & $1700-1960$ \\
\hline Beta-44372 & Zone C & $180 \pm 50$ & 280-Modern & $1670-1960$ \\
\hline Beta-25766 & Zone C & $540 \pm 70$ & $630-490$ & $1320-1460$ \\
\hline Beta-42573 & Zone D & $390 \pm 50$ & $490-320$ & $1460-1630$ \\
\hline Beta-41573 & Zone D & $840 \pm 60$ & $765-670$ & $1185-1280$ \\
\hline Beta-40340 & Zone F & $530 \pm 60$ & $555-490$ & $1395-1460$ \\
\hline Beta-40339 & Zone F & $640 \pm 60$ & $645-540$ & $1305-1410$ \\
\hline Beta-40341 & Zone $\mathrm{F} / \mathrm{H}$ & $670 \pm 60$ & $650-555$ & $1300-1395$ \\
\hline Beta-44373 & Zone $\mathrm{H}$ & $560 \pm 70$ & $630-490$ & $1320-1460$ \\
\hline Beta-33445 & Zone H & $670 \pm 60$ & $650-550$ & $1300-1395$ \\
\hline Beta-25767 & Zone K & $840 \pm 80$ & $790-660$ & $1160-1290$ \\
\hline
\end{tabular}

Note: Samples originally reported in Allen (1994); recalibrated for this paper as per details in Table 1. All analyses were standard radiocarbon determinations, and all material was unidentified wood charcoal.

illa) that are diagnostic for a large number of fish families. Assessment of relative abundances is limited to these five elements so as to not disproportionately advantage a limited number of taxa that have other distinctive and easily identifiable skeletal parts (e.g., scarids and labrids with their hardy and unique pharyngeals, diodons with their numerous body spines, and carangids with their tough diagnostic scutes). Density values (number of identified specimens per cubic meter) also have been calculated (Table 6). Additional quantification measures and analyses are provided in Allen (1992).

\section{Isotope Analysis}

Archaeologists have been utilizing stable isotope analysis for over two decades, and a number of excellent review articles address technical issues and applications (Ambrose and Krigbaum 2003, Katzenberg 2008, LeeThorp 2008). Although relatively new in the Pacific (Leach et al. 1996, 1998, 2003, Ambrose et al. 1997, Pate et al. 2001, Valentin et al. 2006, Beavan-Athfield et al. 2008), several classic studies from elsewhere illustrate how stable isotope analysis of human and animal bones can inform on large-scale subsistence change, as for example, the transition to agriculture (Tauber 1981), the adoption of exotic food crops (e.g., Vogel and van der Merwe 1977, Katzenberg et al. 1995), variation in marine versus terrestrial dietary contributions (e.g., Schoeninger et al. 1983), and trophic level shifts (e.g., Schoeninger and DeNiro 1984). The Aitutaki study utilizes both stable carbon and nitrogen isotopes. Stable isotopes of carbon are useful for discriminating between terrestrial and marine proteins (and their consumers) because marine environments are often enriched in $\delta^{13} \mathrm{C}$ relative to terrestrial ones (Ambrose 1993). Stable isotopes of nitrogen are useful for studying food webs because the $\delta^{15} \mathrm{~N}$ values of bone collagen increase at each successive trophic level, such that carnivores are well differentiated from herbivores in terms of $\delta^{15} \mathrm{~N}$ (Minagawa and Wada 1984, Schoeninger and DeNiro 1984, DeNiro 1987). 


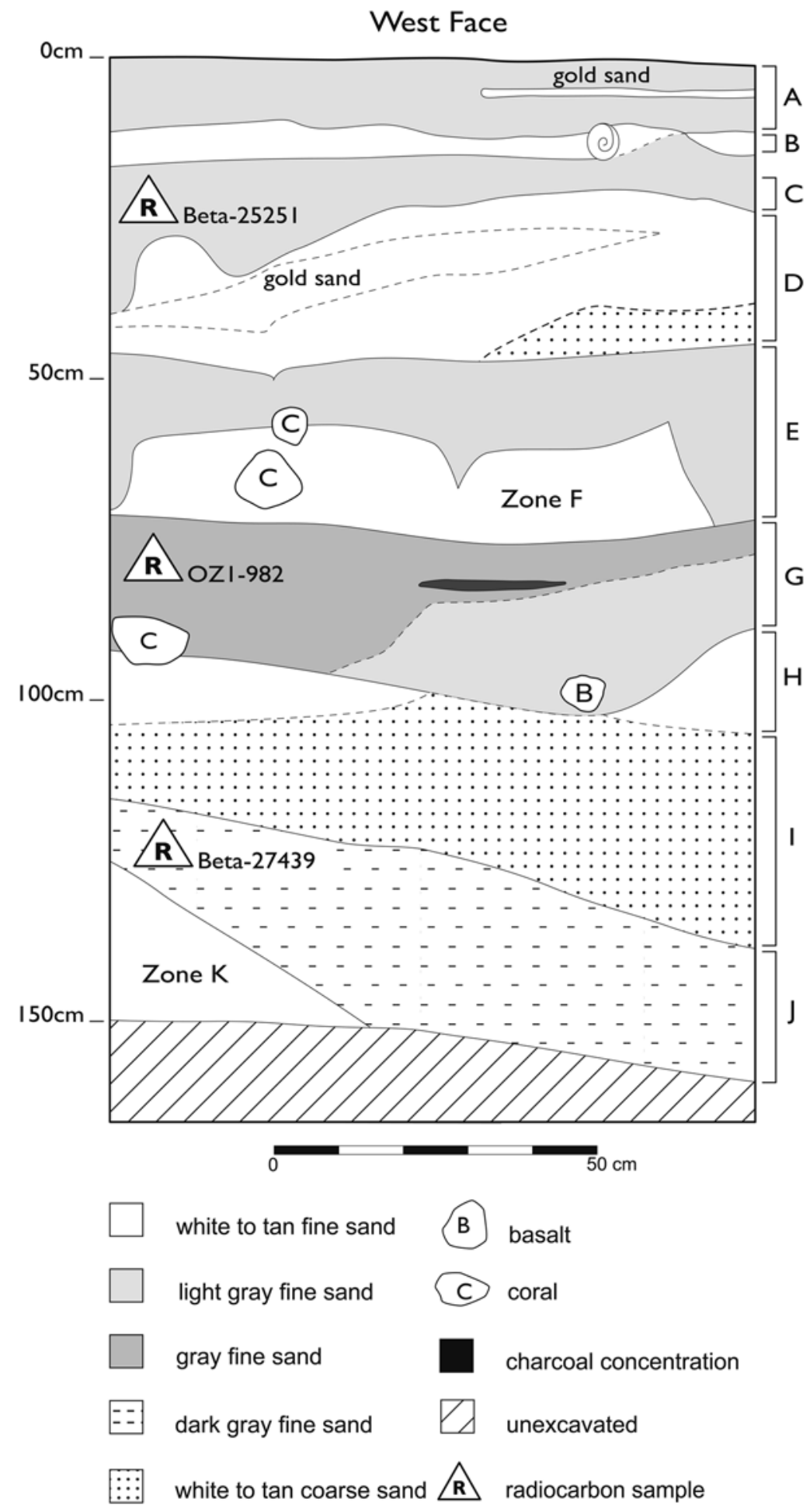

FIgURE 3. The sequence of alternating cultural and storm deposits at Ureia (AIT-10), a stratigraphy that is typical of west-coast localities. 
TABLE 3

Best Age Estimates for Stratigraphic Zones and Time Period Assignments Used in Isotope Comparisons

\begin{tabular}{lccc}
\hline \hline Site and Zone & $\begin{array}{c}\text { Maximal 1 } \sigma \\
\text { Age Range } \\
\text { A.D. }{ }^{a}\end{array}$ & $\begin{array}{c}\text { No. } \\
\text { Radiocarbon } \\
\text { Samples }\end{array}$ & $\begin{array}{c}\text { Time } \\
\text { Period }\end{array}$ \\
\hline Ureia: Zone J & $1210-1430$ & 9 & I \\
Ureia: Zone G & $1220-1440$ & 6 & I \\
Ureia: Zone E & $1220-1390$ & 2 & I \\
Hosea: Zone G & $1300-1430$ & 1 & I \\
$\begin{array}{c}\text { Moturakau: } \\
\text { Zone H }\end{array}$ & $1300-1460$ & 2 & I \\
$\begin{array}{c}\text { Moturakau: } \\
\text { Zones F/H }\end{array}$ & $1300-1460$ & 3 & I \\
$\begin{array}{c}\text { Aretai: Zone G } \\
\text { Moturakau: }\end{array}$ & $1450-1630$ & 1 & II \\
Zone D & $1460-1630$ & 1 & II \\
$\begin{array}{c}\text { Hosea: Zone E } \\
\text { Aretai: Zone E }\end{array}$ & $1490-1670$ & 1 & II \\
$\begin{array}{l}\text { Ureia: Zone C } \\
\text { Moturakau: }\end{array}$ & Post-1660-1670 & 1 & II \\
Zone C & $1670-1960$ & 1 & III \\
Moturakau: & $1700-1960$ & 1 & III \\
Zone B & & 1 & III \\
Moturakau: & $1640-1810$ & 1 & III \\
Zone A & & & \\
\hline
\end{tabular}

${ }^{a}$ Details provided in Tables 1 and 2.

${ }^{b}$ One out-of-sequence determination not included here.

${ }^{c}$ A single sample; this is the $66.2 \%$ probability rather than maximal $1 \sigma$ age range.

For the analysis presented here, samples from 47 individuals were analyzed (Table 7). Differences in specimen age, size, and provenience were used to ensure that each sample represented a distinct individual, as these materials were not associated with discrete burials. The pig and dog samples were essentially food remains, and the human samples consisted of loose teeth, isolated bones, and, in the case of Zone $\mathrm{C}$ at Ureia, loose elements that were most likely displaced from burials (see Bellwood 1978). Both bone (varied elements) and teeth were analyzed. The pre-1850 individuals that form the basis of this analysis include 18 humans, 18 pigs, and 11 dogs (additional samples are reported in Craig [2009]). Twenty-five local plant and animal reference collections were run as well, providing critical information on local background values (Table 8). Using local dietary sources enables much finer-grained and more accurate dietary reconstructions because they take into account variations in geology, rainfall, and crop types. Mainly because of sample size considerations, but also to facilitate intersite comparisons, we have grouped the isotope results into three broad time periods (Table 3).

The Aitutaki samples were processed at the GNS Science National Isotope Centre in Lower Hutt, New Zealand. The analysis protocol was as follows (N. Beavan-Athfield, pers. comm., 2008): bones were brushed to remove burial soil, washed and sonically cleaned in deionized water, and dried in a vacuum oven at $40^{\circ} \mathrm{C}$. Each sample was pulverized using a mortar and pestle to $<450$ $\mu \mathrm{m}$, and demineralized in $0.5 \mathrm{M} \mathrm{HCl}$ while stirring at room temperature for 1-2 hr. Collagen was filtered from the solution and

TABLE 4

Summary of Sample Details

\begin{tabular}{|c|c|c|c|c|c|c|}
\hline \multirow[b]{2}{*}{ Location } & \multirow{2}{*}{$\begin{array}{c}\text { Area } \\
\text { Excavated }\end{array}$} & \multirow{2}{*}{$\begin{array}{l}\text { No. Cultural } \\
\text { Strata }^{a}\end{array}$} & \multirow{2}{*}{$\begin{array}{l}\text { NISP } \\
\text { Fauna }^{b}\end{array}$} & \multicolumn{3}{|c|}{ Isotope Analyses $^{c}$} \\
\hline & & & & Human & Pig & Dog \\
\hline Ureia (AIT-10) & $13 \mathrm{~m}^{2}$ & 5 & 5,449 & 12 & 7 & 5 \\
\hline Hosea (AIT-50) & $7 \mathrm{~m}^{2}$ & 4 & 1,482 & 0 & 4 & 5 \\
\hline Aretai (AIT-49) & $3 \mathrm{~m}^{2}$ & 4 & 407 & 0 & 3 & 1 \\
\hline Moturakau (MR-1) & $16 \mathrm{~m}^{2}$ & 9 & 12,554 & 6 & 4 & 0 \\
\hline
\end{tabular}

${ }^{a}$ Includes modern surface.

${ }^{b}$ Values include materials from modern and noncultural storm layers; unidentified fish bone not included.

${ }^{c}$ Values represent total number of pre-A.D. 1850 individuals. 
TABLE 5

Summary of Vertebrate Fauna (NISP) by Occupation Layer (Data from Allen [1992] ${ }^{a}$ )

\begin{tabular}{|c|c|c|c|c|c|c|c|c|c|c|c|c|c|}
\hline \multirow[b]{2}{*}{ Taxon } & \multicolumn{4}{|c|}{ Ureia } & \multicolumn{2}{|c|}{ Hosea } & \multicolumn{3}{|c|}{ Aretai } & \multicolumn{4}{|c|}{ Moturakau $^{b}$} \\
\hline & $\begin{array}{c}\text { Zone } \\
\text { C }\end{array}$ & $\begin{array}{c}\text { Zone } \\
\mathrm{E}\end{array}$ & $\begin{array}{c}\text { Zone } \\
\text { G }\end{array}$ & $\begin{array}{l}\text { Zone } \\
\text { J }\end{array}$ & $\begin{array}{c}\text { Zone } \\
\mathrm{E}\end{array}$ & $\begin{array}{c}\text { Zone } \\
\text { G }\end{array}$ & $\begin{array}{c}\text { Zone } \\
\text { C }\end{array}$ & $\begin{array}{c}\text { Zone } \\
\mathrm{E}\end{array}$ & $\begin{array}{c}\text { Zone } \\
\text { G }\end{array}$ & $\begin{array}{c}\text { Zone } \\
\text { B }\end{array}$ & $\begin{array}{c}\text { Zone } \\
\mathrm{C}\end{array}$ & $\begin{array}{l}\text { Zone } \\
\text { D }\end{array}$ & $\begin{array}{c}\text { Zones } \\
\text { F/H }\end{array}$ \\
\hline Identified fish ${ }^{c}$ & 839 & 438 & 451 & 127 & 455 & 391 & 17 & 270 & 25 & 1,193 & 1,249 & 2,368 & 4,032 \\
\hline Sea turtle & 5 & 25 & 6 & 12 & 2 & 5 & & & & & & 1 & 1 \\
\hline \multicolumn{14}{|l|}{ Birds } \\
\hline Dendrocygna sp. & 1 & & & & & & & & & & & & \\
\hline Anas superciliosa & 1 & & & & & & & & & & & & \\
\hline Anous stolidus & & & & & & & & & & & & 2 & 4 \\
\hline Egretta sacra & & & & & & & & & & & & & 8 \\
\hline Eudynamis taitensis & & & & & 1 & & & & & 1 & & & \\
\hline Fregata ariel & & & & & & & & & & 1 & & & \\
\hline $\begin{array}{l}\text { Gallus gallus } \\
\text { (chicken) }\end{array}$ & 15 & 19 & 1 & 1 & 3 & 1 & & & & 1 & 1 & 1 & \\
\hline Gygis alba & & & & & & & & & & & & & 1 \\
\hline Numenius tabitiensis & & & & & & & & & & & & 2 & 8 \\
\hline Porzana tabuensis & 11 & 6 & 1 & 2 & 2 & & & & & 4 & & & \\
\hline Sula sula & & & & & & & 1 & & & & & & \\
\hline Vini kublii & & & & & 1 & & & & & & & & \\
\hline Undetermined bird & 13 & 1 & & & & & & & & & 5 & 4 & 2 \\
\hline \multicolumn{14}{|l|}{ Mammals } \\
\hline Homo sapiens & 14 & 9 & & 3 & 2 & & & & & & & & \\
\hline Sus scrofa (pig) & 25 & 17 & 21 & 3 & 17 & 9 & & 12 & 5 & & 1 & & 6 \\
\hline $\begin{array}{l}\text { Canis familiaris } \\
\quad(\mathrm{dog})\end{array}$ & 440 & 15 & 9 & & 6 & 2 & 1 & 3 & & & & & \\
\hline $\begin{array}{l}\text { Rattus exulans } \\
\quad \text { (Polynesian Rat) }\end{array}$ & 202 & 662 & 241 & 122 & 79 & 44 & 3 & 7 & 4 & 69 & 116 & 74 & 23 \\
\hline Pteropus tonganus & & & & & 2 & & & & & & & & \\
\hline Sea mammal & & 3 & & & & 1 & & & & & & & \\
\hline $\begin{array}{l}\text { Unidentified } \\
\text { mammal }\end{array}$ & 53 & 41 & 18 & 26 & 20 & 11 & 1 & 28 & 7 & 1 & 2 & 7 & 11 \\
\hline $\begin{array}{l}\text { Unidentified } \\
\text { vertebrate }\end{array}$ & 28 & 32 & 21 & 1 & 99 & 34 & & 12 & 1 & 6 & 4 & 14 & 21 \\
\hline
\end{tabular}

${ }^{a}$ Data from modern layers and noncultural storm deposits are available in Allen (1992).

$b$ Shelter B only.

$c$ These values are total NISP, including both the five paired jaw elements and all other identified elements. See Allen [1992, 2002] for more detailed information on the recovered taxa.

gelatinized with $0.01 \mathrm{M} \mathrm{HCl}$ in a nitrogen atmosphere at $100^{\circ} \mathrm{C}$ for $16 \mathrm{hr}$. The gelatin was double-filtered (Whatman GF/C and 0.45 $\mu \mathrm{m}$ Acrodisc filters) and lyophilized to determine yields.

Samples were run in an elemental analyser (ANCA-SL) in continuous flow mode, interfaced to a mass spectrometer (Europa Scientific Geo 20/20). Carbon and nitrogen isotopes were analyzed simultaneously from an average $1.5 \mathrm{mg}$ of sample. The $\mathrm{CO}^{2}$ and nitrogen gases were resolved using chromatographic separation on a gas chromatograph column at $85^{\circ} \mathrm{C}$ and analyzed for $\delta^{13} \mathrm{C}, \delta^{15} \mathrm{~N}$, $\% \mathrm{C}$ and $\% \mathrm{~N}$, and $\mathrm{C} / \mathrm{N}$ ratios. Measurement error values are $\pm 0.1 \%$ for carbon and $\pm 0.3 \%$ or better for nitrogen.

The most common way of determining whether a sample has undergone diagenetic alteration of collagen is to examine the $\mathrm{C}: \mathrm{N}$ ratio. In fresh bone the ratio is typically between 2.9 and 3.6 (DeNiro 1985:807), and it has been shown that samples with $\mathrm{C}: \mathrm{N}$ ratios outside that range can produce unreliable results, although some analysts will consider values outside that range (DeNiro 1985, 
TABLE 6

Density (NISP $/ \mathrm{m}^{3}$ ) of Nonfish Vertebrate Fauna by Zone (from Allen 1992)

\begin{tabular}{|c|c|c|c|c|c|c|c|c|c|c|c|c|}
\hline \multirow[b]{2}{*}{ Taxa } & \multicolumn{3}{|c|}{ Ureia } & \multicolumn{2}{|c|}{ Hosea } & \multicolumn{3}{|c|}{ Aretai } & \multicolumn{4}{|c|}{ Moturakau $^{b}$} \\
\hline & $\mathrm{C}$ & $\mathrm{E}$ & G & $\mathrm{E}$ & G & $\mathrm{C}$ & $\mathrm{E}$ & G & B & $\mathrm{C}$ & $\mathrm{D}$ & $\mathrm{F} / \mathrm{H}$ \\
\hline \multicolumn{13}{|l|}{ Introduced taxa } \\
\hline Chicken & 11.7 & 8.5 & 1.3 & 1.6 & 0.9 & & & & 0.5 & 0.6 & 0.8 & \\
\hline Pig & 8.8 & 11.6 & 26.8 & 9.0 & 8.1 & & 9.0 & 17.9 & & 0.6 & & 9.2 \\
\hline Dog & $48.1^{a}$ & 6.9 & 10.2 & 3.2 & 1.8 & 7.3 & 2.3 & & & & & \\
\hline Rattus exulans & 70.0 & 134.9 & 142.7 & 42.0 & 39.7 & 21.8 & 5.3 & 14.3 & 37.7 & 67.7 & 57.6 & 20.6 \\
\hline Human & & 1.1 & & 1.1 & & & & & & & & \\
\hline Subtotal & 138.6 & 163.0 & 181.0 & 56.9 & 50.5 & 29.1 & 16.6 & 32.2 & 38.2 & 68.9 & 58.4 & 29.8 \\
\hline Indigenous taxa & & & & & & & & & & & & \\
\hline Wild birds & 4.4 & 0.5 & & 2.1 & & 7.3 & & & 2.7 & 0.6 & 3.1 & 28.3 \\
\hline $\begin{array}{l}\text { Pteropus } \\
\text { Sea mammal }\end{array}$ & & & & 1.1 & & & & & & & & \\
\hline Sea mammal & & 1.1 & & & 0.9 & & & & & & & \\
\hline Sea turtle & 2.9 & 12.2 & 2.5 & 1.1 & 4.5 & & & & & & 0.8 & 0.9 \\
\hline Subtotal & 7.3 & 13.8 & 2.5 & 4.3 & 5.4 & 7.3 & 0 & 0 & 2.7 & 0.6 & 3.9 & 29.2 \\
\hline \multicolumn{13}{|l|}{ Undetermined } \\
\hline Unid. mammal & 2.9 & 3.2 & & 10.6 & 9.9 & 7.3 & 21.1 & 25.0 & 0.5 & 1.2 & 5.5 & 19.4 \\
\hline Unid. vertebrate & 49.6 & 28.0 & 31.9 & 52.6 & 30.7 & & 9.0 & 3.6 & 3.3 & 5.2 & 14 & 27.1 \\
\hline Total & 198.4 & 208.0 & 215.4 & 124.4 & 96.5 & 43.7 & 46.7 & 60.8 & 44.7 & 75.9 & 81.8 & 105.5 \\
\hline
\end{tabular}

${ }^{a}$ Excludes juvenile dog burial from feature 1 of Unit 13.

${ }^{b}$ Shelter B only.

Schoeninger 1989, Ambrose 1990, Coltrain et al. 2004, Bösl et al. 2006). DeNiro's (1985) more conservative range is used herein because this was the first isotopic analysis undertaken in the Cook Islands, and the potential range of variation in $\mathrm{C}: \mathrm{N}$ ratios was unknown. Samples outside this range were not included in the analysis presented here.

A small number of analyses run on bone and teeth from the same individuals suggested that the two material types provide similar results, all other factors being constant. Samples also were evaluated for the possibility of elevated $\delta^{15} \mathrm{~N}$ values related to breast-feeding (Fogel et al. 1997, Larsen 1997, Herring et al. 1998); all of the Aitutaki samples reported here appear to postdate weaning (see details in Craig [2009]).

\section{Comparative Considerations}

Archaeofaunal assemblages and stable isotopic signatures are the result of quite different formation processes and thus have varied analytical sensitivities. Archaeofaunal assem- blages are time-averaged records, the result of numerous activities by groups of unknown size and composition, often accumulated over extended periods of time; as such they offer information on the broad patterns of subsistence change. Stable isotopes, alternatively, record the diets of individuals and relate to quite delimited periods of time, decadal or in some cases only 2 to 3 years, depending on the life span of an animal. Although both archaeofaunal remains and bone chemistry values can be affected by postdepositional processes, archaeological recovery techniques, and various analytical decisions (sampling, quantification, etc.), these differ for each record and are largely independent from one another. Thus findings from one usefully serve as a check on the interpretive results of the other. The two records also provide quite different kinds of information. Polynesian faunal assemblages typically best reflect wild resource use, with animal domesticates often being poorly represented. Stable isotopes, in contrast, provide information on both plant and animal components of the diet, albeit in a more coarse-grained fashion. 
TABLE 7

Isotope Values by Sample (Data from Craig [2009])

\begin{tabular}{|c|c|c|c|c|c|}
\hline Accession No. ${ }^{a}$ & Site & $\begin{array}{l}\text { Analytic } \\
\text { Zone }\end{array}$ & $\delta^{13} \mathrm{C}$ & $\delta^{15} \mathrm{~N}$ & $\begin{array}{l}\mathrm{C}: \mathrm{N} \\
\text { Ratio }\end{array}$ \\
\hline \multicolumn{6}{|l|}{ Human } \\
\hline 98.1 & AIT-10 & $\mathrm{C}$ & -15.4 & 11.9 & 3.3 \\
\hline 161.1 & $\mathrm{AIT}-10$ & $\mathrm{C}$ & -16.1 & 11.5 & 3.2 \\
\hline BEL.2 & AIT-10 & $\mathrm{C}$ & -16.7 & 10.4 & 3.3 \\
\hline BEL.3 & AIT-10 & $\mathrm{C}$ & -16.9 & 10.3 & 3.3 \\
\hline BEL. 19.3 & AIT-10 & $\mathrm{C}$ & -14.2 & 11.3 & 3.3 \\
\hline BEL.21.3 & AIT-10 & $\mathrm{C}$ & -13.6 & 11.8 & 3.3 \\
\hline BEL. 25 & AIT-10 & $\mathrm{C}$ & -15.2 & 11.1 & 3.2 \\
\hline BEL.26 & AIT-10 & $\mathrm{C}$ & -13.5 & 11.5 & 3.2 \\
\hline BEL.31 & AIT-10 & $\mathrm{C}$ & -16.7 & 10.8 & 3.3 \\
\hline 303.1 & AIT-10 & G & -14.6 & 11.0 & 3.2 \\
\hline 195.1 & AIT-10 & $\mathrm{J}$ & -14.5 & 11.9 & 3.2 \\
\hline 194.1 & AIT-10 & $\mathrm{J}$ & -14.9 & 12.0 & 3.3 \\
\hline 153.1 & MR-1 & $\mathrm{C}$ & -16.8 & 12.9 & 3.3 \\
\hline 45 & MR-1 & $\mathrm{E}$ & -17.9 & 10.6 & 3.2 \\
\hline 89.3 & MR-1 & $\mathrm{E}$ & -16.6 & 12.6 & 3.1 \\
\hline 121.1 & MR-1 & $\mathrm{E}$ & -15.3 & 12.9 & 3.1 \\
\hline 136 & MR-1 & $\mathrm{F}$ & -17.9 & 11.2 & 3.2 \\
\hline 138 & MR-1 & $\mathrm{H}$ & -17.6 & 11.2 & 3.2 \\
\hline \multicolumn{6}{|l|}{ Pig } \\
\hline 276.1 & AIT-10 & $\mathrm{C} / \mathrm{D}^{b}$ & -17.5 & 9.8 & 3.3 \\
\hline 276.2 & AIT-10 & $\mathrm{C} / \mathrm{D}^{b}$ & -18.0 & 9.0 & 3.4 \\
\hline 276.3 & AIT-10 & $\mathrm{C} / \mathrm{D}^{b}$ & -17.2 & 10.6 & 3.3 \\
\hline 115.2 & AIT-10 & $\mathrm{E}$ & -18.6 & 11.7 & 3.3 \\
\hline 279.1 & AIT-10 & $\mathrm{E}$ & -16.3 & 10.1 & 3.5 \\
\hline 117.1 & AIT-10 & G & -16.3 & 12.2 & 3.3 \\
\hline 285.3 & AIT-10 & G & -17.8 & 15.3 & 3.4 \\
\hline 12.1 & AIT-49 & $\mathrm{E}$ & -18.3 & 12.8 & 3.0 \\
\hline 20.1 & AIT-49 & $\mathrm{E}$ & -18.8 & 10.9 & 3.1 \\
\hline 19.2 & AIT-49 & G & -16.6 & 9.9 & 3.0 \\
\hline 8.1 & AIT-50 & $\mathrm{E}$ & -16.5 & 14.6 & 3.0 \\
\hline 29.1 & AIT-50 & $\mathrm{E}$ & -17 & 11.6 & 3.4 \\
\hline 44.3 & AIT-50 & $\mathrm{E}$ & -18.4 & 11.6 & 3.2 \\
\hline 9.1 & AIT-50 & $\mathrm{G}$ & -14.3 & 10.9 & 3.3 \\
\hline 43.1 & MR-1 & $\mathrm{D}$ & -16.4 & 10.5 & 3.1 \\
\hline 90.7 & MR-1 & $\mathrm{F}$ & -17.5 & 11.1 & 3.2 \\
\hline 67.1 & MR-1 & $\mathrm{F}$ & -17.1 & 14.6 & 3.0 \\
\hline 235.1 & MR-1 & $\mathrm{H}$ & -16.9 & 11.5 & 3.1 \\
\hline \multicolumn{6}{|l|}{ Dog } \\
\hline 240.1 & AIT-10 & $\mathrm{C} / \mathrm{D}^{b}$ & -10.4 & 12.1 & 3.3 \\
\hline 226.1 & AIT-10 & $\mathrm{E}$ & -14.3 & 11.1 & 3.3 \\
\hline 226.2 & AIT-10 & $\mathrm{E}$ & -13.6 & 12.4 & 3.2 \\
\hline 102.2 & AIT-10 & G & -13.7 & 11.9 & 3.2 \\
\hline 285.2 & AIT-10 & G & -11.8 & 11.3 & 3.0 \\
\hline 12.2 & AIT-49 & $\mathrm{E}$ & -17.3 & 13.0 & 3.4 \\
\hline 2.2 & AIT-50 & $\mathrm{E}$ & -14.3 & 14.9 & 2.9 \\
\hline 37.1 & AIT-50 & $\mathrm{E}$ & -12.6 & 11.1 & 3.5 \\
\hline 44.1 & AIT-50 & E feature 2 & -14.4 & 12.5 & 3.3 \\
\hline 44.2 & AIT-50 & E feature 2 & -17.4 & 11 & 3.2 \\
\hline 23.1 & AIT-50 & G & -14.7 & 14.6 & 2.9 \\
\hline
\end{tabular}

Note: Machine error values are $\pm 0.1 \%$ for carbon and $\pm 0.3 \%$ or better for nitrogen. ${ }^{a}$ Samples prefaced by BEL were excavated by Peter Bellwood (1978).

${ }^{b}$ Zones $\mathrm{C}$ and $\mathrm{D}$ were not clearly differentiated in the unit these samples came from. 
TABLE 8

Stable Isotope Values for Aitutaki Reference Samples (Data from Craig 2009)

\begin{tabular}{|c|c|c|c|c|}
\hline Common Name & Scientific Name & $\delta^{13} \mathrm{C}$ & $\delta^{15} \mathrm{~N}$ & C:N Ratio \\
\hline Coconut palm & Cocos nucifera & -22.38 & 8.3 & 43.2 \\
\hline Taro corm & Colocasia esculenta & -26.80 & 3.9 & 154.0 \\
\hline Taro corm & Colocasia esculenta & -25.50 & 1.9 & 59.9 \\
\hline Taro leaf & Colocasia esculenta & -24.67 & 10.85 & 14.4 \\
\hline Atoll taro corm & Cyrtosperma chamissonis & -29.35 & 7.42 & 9.1 \\
\hline Atoll taro leaf & Cyrtosperma chamissonis & -26.1 & 6.7 & 11.8 \\
\hline Banana & Musa $\times$ paradisiaca & -23.17 & 6.03 & 61.5 \\
\hline Pacific arrowroot & Tacca leontopetaloides & -23.66 & 9.14 & 23.0 \\
\hline Breadfruit & Artocarpus altilis & -28.2 & 8.1 & 40.2 \\
\hline Sweet potato & Ipomoea batatas & -26.79 & 8.5 & 55.0 \\
\hline Indian mulberry & Morinda citrifolia & -26.52 & 2.49 & 41.6 \\
\hline Malay apple & Syzygium malaccense & -28.26 & 7.37 & 48.3 \\
\hline $\mathrm{Ti}$ & Cordyline fruticosa & -26.38 & 5.46 & 25.1 \\
\hline Native pigweed & Portulaca lutea & -12.42 & 8.37 & 11.5 \\
\hline Blotcheye soldierfish & Myripristis cf. amaena & -16.52 & 13.99 & 3.6 \\
\hline Golden-lined sea perch & Gnathodentex aureolineatus & -14.03 & 12.82 & 3.6 \\
\hline Blue-spotted grouper & Cephalopholis argus & -12.81 & 13.68 & 3.1 \\
\hline Honeycomb sea bass & Epinephelus merra & -12.77 & 13.15 & 3.1 \\
\hline Red-spotted surgeonfish & Acanthurus achilles & -11.74 & 9.83 & 3.4 \\
\hline Convict tang & Acanthurus triostegus & -10.25 & 8.56 & 3.1 \\
\hline Black durgon & Melichthys niger & -12.52 & 11.00 & 3.2 \\
\hline Chub cf. Highfin & Kyphosus cf. cinerascens & -16.50 & 11.11 & 3.1 \\
\hline Damselfish & Pomacentridae & -17.13 & 11.08 & 3.0 \\
\hline Parrotfish cf. Bullethead & Chlorurus cf. sordidus & -11.15 & 10.35 & 3.2 \\
\hline Parrotfish cf. minifin & Scarus cf. altipinnis & -10.40 & 7.64 & 3.2 \\
\hline
\end{tabular}

Note: Machine error values are $\pm 0.1 \%$ for carbon and $\pm 0.3 \%$ or better for nitrogen.

Stable carbon isotopes specifically track the relative contribution of four broad categories of plants that have different $\delta^{13} \mathrm{C}$ carbon signatures $\left(C_{3}, C_{4}, C A M\right.$, and marine taxa such as seaweeds) (see Figure 5). Stable nitrogen isotopes inform on the source of dietary protein, being especially helpful in identifying the importance of marine versus terrestrial food sources, as well as the trophic levels from which the consumed foods derived. Stable isotopes also allow us to extend our understanding of island subsistence economies to species directly managed by humans (domesticates), as well as those loosely associated with anthropogenic environments (commensals). We further explore these methodological potentials and challenges in the context of the Aitutaki case study.

All statistical analyses were carried out using SPSS 14.0 and 16.0. When two or more groups of data were being tested for differences, the data were evaluated for normal dis- tribution using Levene's Test of Equality of Error Variances. If the $P$ value was not significant (greater than .05) then the populations were considered normally distributed and Student's $t$-test was used to compare the variation between two groups, and analysis of variance (ANOVA) was used to compare three or more groups. A $P$ value of .05 or less was considered significant. Where ANOVA indicated significant differences between groups of three or more, post-hoc analysis of pairwise comparisons was done using Tukey's Honestly Significant Differences (HSD) test to determine which particular groups were significantly different. However, if the Levene's test was significant (less than $.05)$ then the data were not normally distributed and nonparametric tests were used. The Mann-Whitney $U$ test was used for two groups, and the Kruskal-Wallis analysis of variance for groups of three or more. P values of .05 or less were considered significant. 
RESULTS

\section{Colonial or Established Community?}

As one of the older localities of human occupation in the southern Cook Islands, a key question has been whether Aitutaki's earliest cultural layers represent colonizing populations or established groups. Steadman (1991) argued that the limited range of native bird species and their low abundances were not reflective of a pristine avifauna (see also Table 5). Similarly, sea turtles, another vulnerable taxon, are also limited at these sites. Shellfish from the earliest levels were neither taxonomically distinctive nor particularly large, as might be expected for a previously unexploited resource. In contrast, domesticates (pig, dog, and chicken) and the Polynesian Rat (Rattus exulans) are represented throughout the sequence, albeit in modest numbers.

There is, however, variability in domesticates and the commensal rat. The marked increase in dog NISP in the late prehistoric layer (Zone C) of Ureia is the result of a single dog burial. The increase in chicken at this same locality, however, appears to reflect local dietary changes. The very high rat abundances at Ureia are also notable, a species that is not nearly so well represented in the other sites. Missionary John Williams (1837:154) commented on Aitutaki's "astonishing" rat populations such that "we never sat down to a meal without two or more persons to keep them off the table." Nevertheless, neither he nor Buck (1927) noted that they were eaten, and both Allen (1992:390) and Colman (1998) in his more systematic study failed to find evidence that was consistent with rat consumption. Contrasting with the mainland sites, the Moturakau assemblage has a more diverse bird assemblage, which is not surprising in light of the islet's more temporary usage and its better access to other uninhabited islets where birds might nest. These patterns aside, the vertebrate fauna as a whole suggests a landscape that had already experienced substantial human modification, a view supported by wood charcoal and land snail evidence as well (Allen 1992, Allen and Wallace 2007).
The stable isotope results broadly corroborate the vertebrate record and offer the first insights into the contribution of plant foods to the local diet (Table 7, Figures 4 and 5). This latter facet of the isotope results takes on added importance here because there is little field evidence for past agricultural activities on the island (cf. Bellwood 1978). Given what is known about the indigenous Polynesian flora, both here and elsewhere (e.g., Fosberg 1975, Whistler 1991), it is unlikely that the island supported native carbohydrates of consequence in the past. We therefore assume that the ingested plant proteins were largely derived from cultivated species. Most of the introduced Polynesian domesticates, including taro, yams, banana, breadfruit, and sweet potato have $\mathrm{C}_{3}$ photosynthetic pathways, consistent with the isotope findings. The only known $\mathrm{C}_{4}$ domesticate is sugarcane, although at least one incidental wild food source, Portulaca lutea, is a CAM/C $\mathrm{C}_{4}$ plant (Guralnick et al. 2002).

As illustrated in Figures 4 and 5, the Aitutaki consumers occupy an intermediate position relative to background terrestrial and marine food webs, suggesting that both sources were important (note that in each case archaeological consumer values have been adjusted for trophic fractionation, specifically -1 per mil for carbon, -3 per mil for nitrogen). This pattern holds not only for the humans, but also generally for the Aitutaki pigs and dogs. Notably, these values may underestimate the importance of plant foods on Aitutaki, given that studies of populations with high-protein diets indicate that dietary carbohydrates are considerably underrepresented in the isotopic composition of bone collagen (Ambrose 1993:82, Ambrose and Norr 1993). The stability of $C_{3}$ plant contributions over time, coupled with the intermediate position of these individuals relative to marine and terrestrial food sources, is consistent with the idea that the earliest occupation on Aitutaki represents a settled community with an established agroeconomy. By way of contrast, populations consuming large amounts of animal protein (e.g., birds, turtles, etc.), as was typical for early Polynesian colonists elsewhere, would be expected to have enriched stable carbon isotope values. 


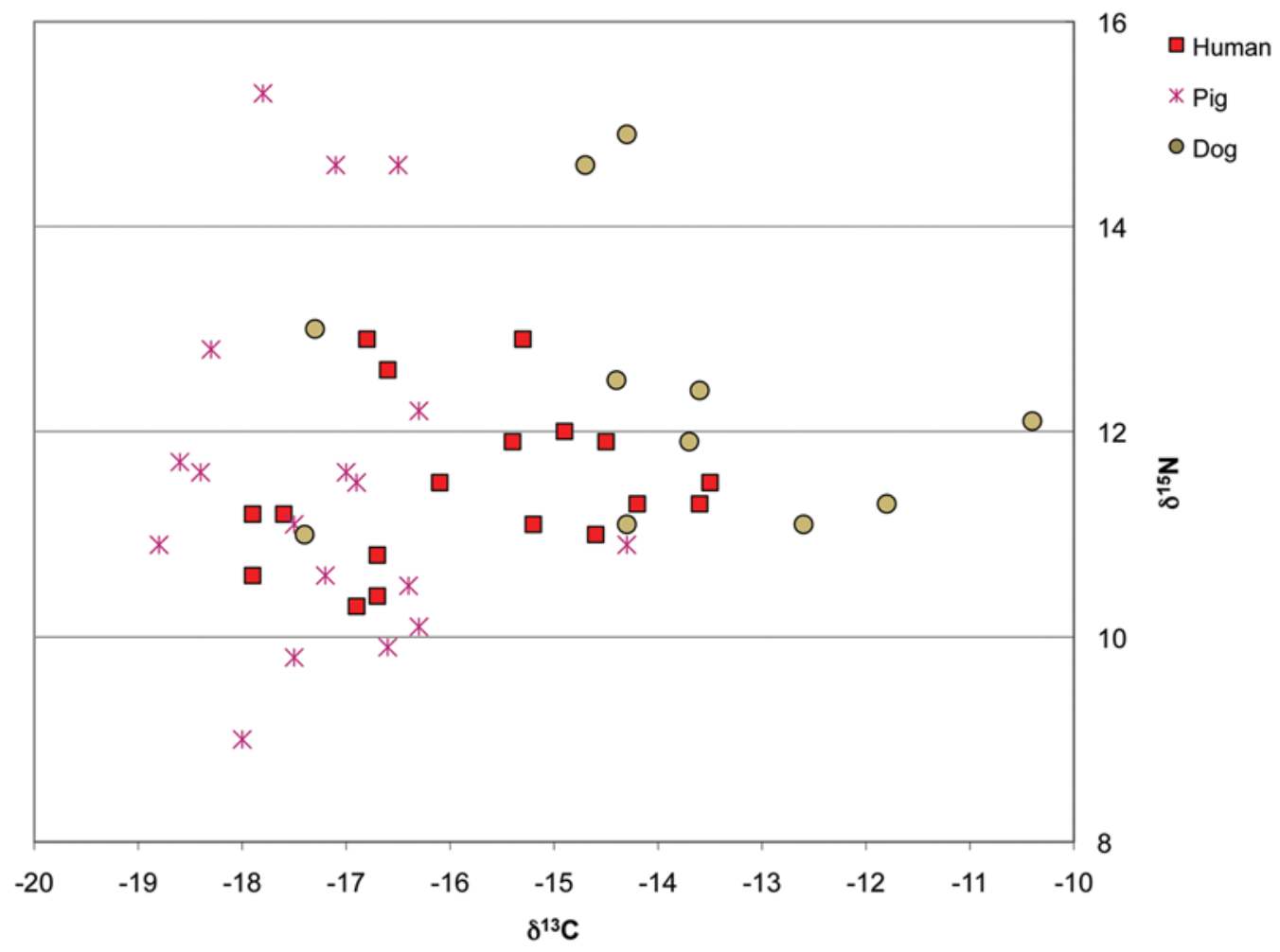

FIGURE 4. Isotopic values of archaeological human, pig, and dog samples.

\section{Declines in Marine Protein}

Although no dramatic dietary shifts are indicated by the isotopic evidence, subtle change is apparent, consistent with certain archaeofaunal findings. At the mainland site of Ureia, the time-averaged faunal assemblages, partitioned by abrupt sedimentary events (i.e., storms), evidence a marked decline in fish bone (Figure 6). This decline (NISP per cubic meter) is first apparent between Zone $G$ and Zone E, two strata that both date to about the fourteenth century A.D. Similar changes are also registered at the Hosea site, a few kilometers to the north, between the fourteenth and sixteenth centuries A.D. At both sites the decline in fish bone deposition continues into the historic period.

There are a number of possible explana- tions for this trend. Declines in fish bone remains could relate to changes in the rates of sediment accumulation. To evaluate this idea, age-depth relations were explored using the Ureia stratigraphic record and 27 radiocarbon determinations from this site. The results indicate that initially sedimentation rates were high, as the nearshore beach berm developed. But, as sea level fall continued and the beach ridge grew, the rate of sediment accumulation slowed. Fish bone density values, in contrast, were initially high and decreased over time. The density of nonfish vertebrates ( $\mathrm{Ta}$ ble 6), however, was essentially stable between Zone G (215.4 NISP/m $\left.\mathrm{m}^{3}\right)$ and Zone E $\left(208 \mathrm{NISP} / \mathrm{m}^{3}\right)$, as was the case for shellfish (Allen 1992). These findings indicate that changing sedimentation rates cannot explain long-term trends in fish bone density. 


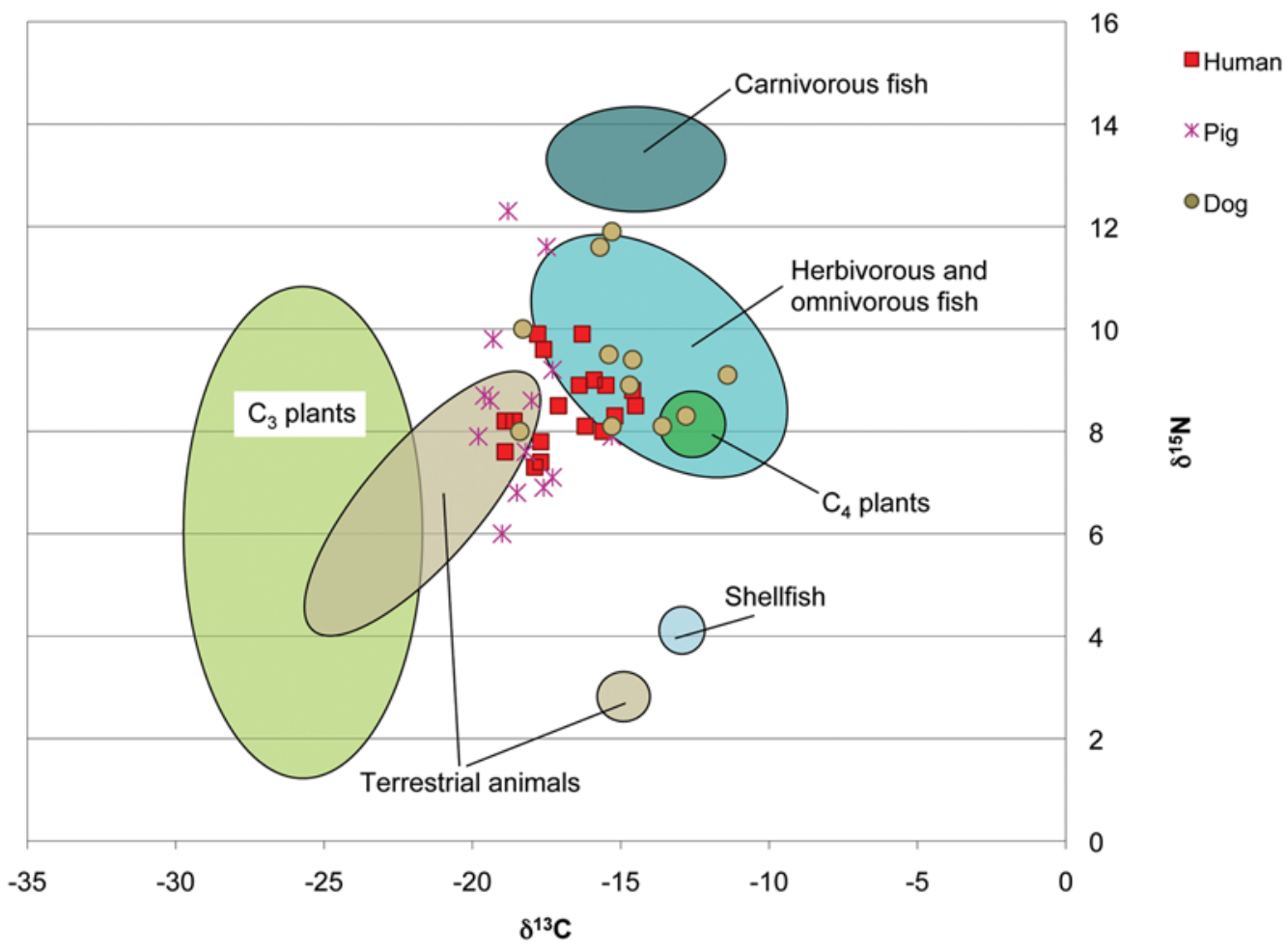

Figure 5. Archaeological human, pig, and dog isotope results shown in relation to potential dietary sources. Archaeological consumer values have been adjusted for trophic fractionation, specifically $-1 \%$ for carbon and $-3 \%$ for nitrogen.

A second hypothesis was that human harvesting of reef resources had an adverse impact on nearshore fish populations, leading to lower capture and consumption rates. This possibility was evaluated by Allen (2002), who found modest support for resource depression at the permanent mainland site of Ureia. The evidence included changes in the proportion of large-bodied taxa over time and possibly a shift in patch use but no clear size changes within preferred species and no change in fish diversity. At the specialized campsite of Moturakau (Figure 7), in contrast, there was little evidence for resource depression but dramatic declines in fish bone over time. As a whole, the evidence suggests that although some changes seen at Ureia could reflect harvesting pressures, declines in fish bone deposition across Aitutaki Island as a whole probably do not; this is not surprising given the small size of the island and the comparatively extensive shallow-water environment.

A third possibility was that declines in fish bone could reflect changes in cultural patterns of deposition, as for example: (1) new patterns of off-site refuse disposal; (2) changes in animal feeding practices, with scraps systematically being collected for domestics; or (3) a decline in the local human population such that sites on the coast were used less intensively and thus less bone was deposited at these localities. Although none of these possibilities could be conclusively eliminated, the marked pattern of fish bone decline at the offshore fishing camp of Moturakau indicated that this was an islandwide phenomenon and therefore probably unrelated to the rise of novel discard practices. 


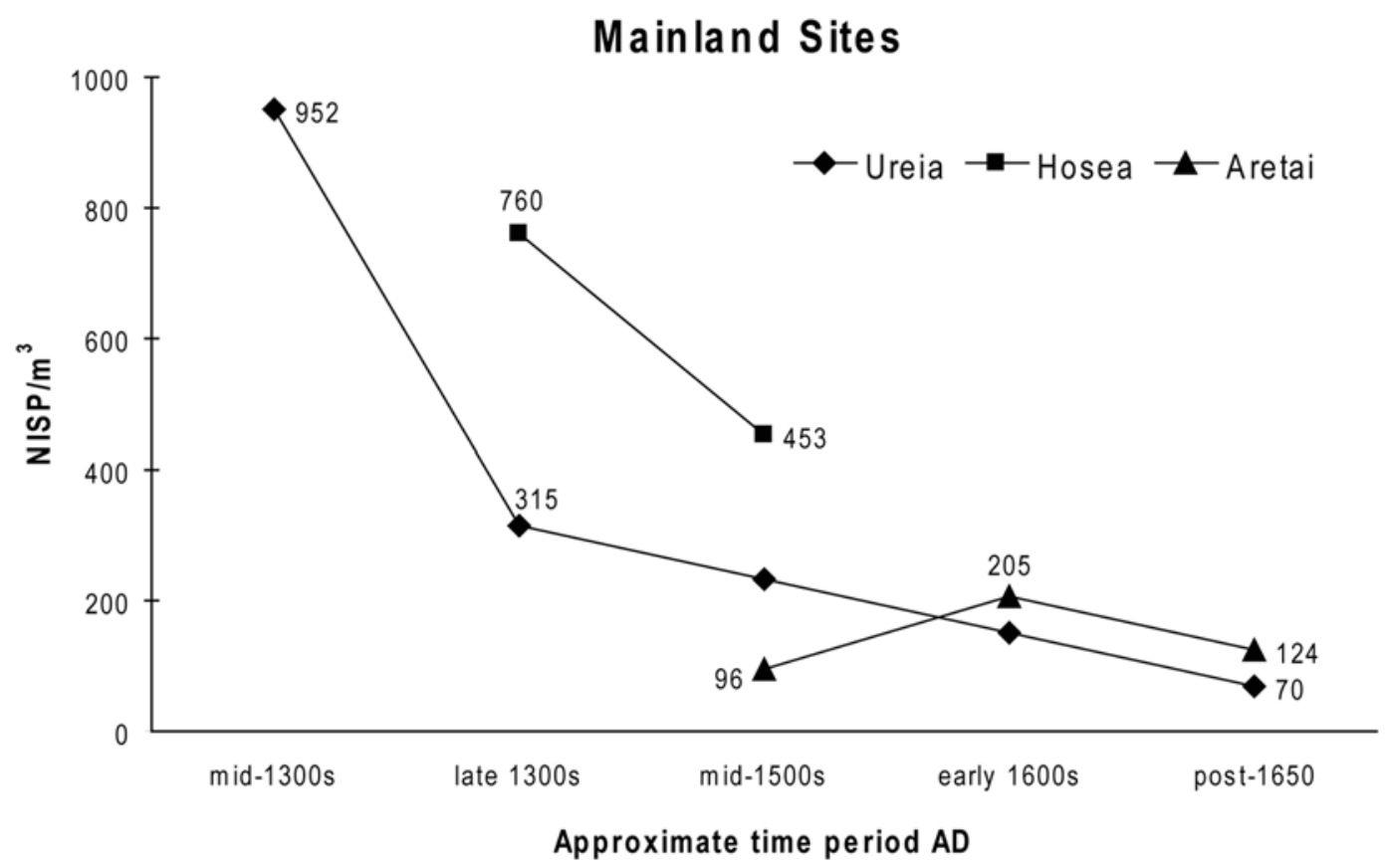

Figure 6. Declines in density of fish bone at the three mainland sites (modified from Allen [2002]). Time periods are approximate and based on both stratigraphic relations and radiocarbon determinations (see Tables 1 and 2 for details).

\section{Moturakau Rockshelter}

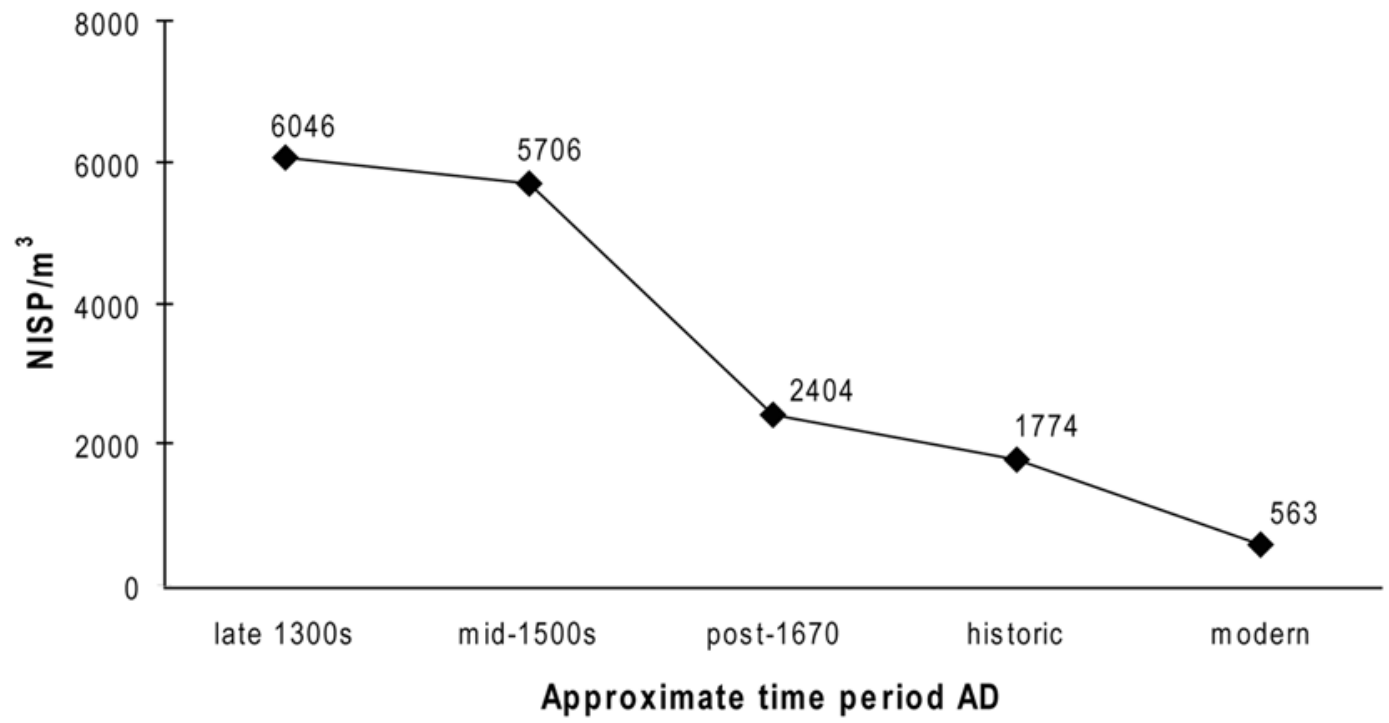

Figure 7. Declines in density of fish bone at Moturakau Rockshelter, Shelter B. Time periods are approximate and based on both stratigraphic relations and radiocarbon determinations (see Table 2 for details). 
A final hypothesis is suggested by the sedimentary record from Ureia and other west coast localities (Figure 3). The series of storm deposits recorded there point to climatic instability, which in all likelihood affected reef resources to some degree. The dense radiocarbon chronology now assembled for Ureia allows us to place at least two, and possibly three, disruptive storm events in the fourteenth century A.D. (further radiocarbon dates are pending). In the case of Zone $\mathrm{J}$, the storm activity (designated Zone J2) nearly obliterated the cultural deposit (Zone J1), placing most of the cultural materials in secondary context. After the storm event that buried Zone E, the area was abandoned and not resettled until shortly before Western contact (Zone C).

Stable isotopes offer an independent means by which to further evaluate the alternatives we have outlined here. Comparison of the mean population values for both pigs and dogs of Periods I and II indicates that those of Period II are more depleted in $\delta^{13} \mathrm{C}$ than those of Period I, with shifts in both the mean values and the ranges (Table 9). These findings hint at a shift away from marine protein resources and toward terrestrial ones, a result that fits comfortably with the vertebrate evidence and supports the interpretation of dietary change rather than altered disposal patterns. Unfortunately, the stable isotope results are not statistically significant, either for individual species or for the assemblage as a whole.

Why is this the case? Problematically, there are differences in the dietary patterns of the three species that hold across all three time periods (Figure 4). For example, pigs are typically more depleted in $\delta^{13} \mathrm{C}$ than dogs. Further, the three species are not uniformly represented in the three time periods, with dogs mainly limited to Periods I and II, and humans absent from Period II. These issues make it difficult to unambiguously separate temporal changes from intraspecific dietary preferences. As a result, changes in $\delta^{13} \mathrm{C}$ are potentially masked when the samples are considered as a group. At the same time, the individual sample sizes are too small to place much confidence in the suggested intraspecies $\delta^{13} \mathrm{C}$ shifts, despite the consistent pattern of $\delta^{13} \mathrm{C}$ enrichment between Periods I and II.

If fish resources were declining, as suggested by the archaeofaunal evidence, then why is the shift in $\delta^{13} \mathrm{C}$ not more marked? One possibility is that the loss of fish resources was offset by increased use of shellfish. Had this been the case, $\delta^{13} \mathrm{C}$ values would have remained relatively stable while $\delta^{15} \mathrm{~N}$ values declined, both conditions that occur (see next section). However, the shellfish records from Ureia and Hosea do not support this interpretation because there is no marked increase in shellfish deposition at either site (see Allen 1992). Similarly, at Moturakau

TABLE 9

Summary of Changing $\delta^{13} \mathrm{C}$ Values across All Sites (Period III Values Shown for Comparison Only)

\begin{tabular}{lllll}
\hline \hline Taxon & \multicolumn{1}{c}{$\begin{array}{c}\text { Period I } \\
\text { Mean } \delta^{13} \mathrm{C}\end{array}$} & \multicolumn{1}{c}{$\begin{array}{c}\text { Period II } \\
\text { Mean } \delta^{13} \mathrm{C}\end{array}$} & ANOVA & $\begin{array}{c}\text { Period III } \\
\text { Mean } \delta^{13} \mathrm{C}\end{array}$ \\
\hline Pig & $-16.9(n=8)$ & $-17.4(n=7)$ & $F=.906$ & $-17.6(n=3)$ \\
& Range: -18.6 to -14.3 & Range: -18.8 to -16.4 & $\mathrm{df}=1,13$ & $P=.359$ \\
Dog & SD 1.2851 & SD 1.0307 & $F=2.21$ & $-10.4(n=1)$ \\
& $-13.6(n=5)$ & $-15.2(n=5)$ & $\mathrm{df}=1,8$ & $P=.175$ \\
All taxa & Range: -14.7 to -11.8 & Range: -17.4 to -12.6 & $F=1.053$ & $-15.6(n=14)$ \\
& SD 1.1122 & SD 2.0893 & df $=1,31$ & \\
& $-15.8(n=21)$ & $-16.5(n=12)$ & & \\
& Range: -18.6 to -11.8 & Range: -18.8 to -12.6 & &
\end{tabular}


there are no clear trends in the density of shellfish remains over time. Further, had nearshore marine environments been damaged by tropical cyclones, as suggested earlier, it is likely that shellfish also would have been adversely affected. It remains unclear why the vertebrate fauna and isotopic evidence are not more closely aligned. We think it likely that interspecific variability, coupled with the uneven temporal distribution of the three species and small sample sizes generally, are masking patterns of change in the isotopic record.

\section{Declines in Consumption of Marine Carnivores}

Other dietary changes took place after ca. A.D. 1450. The robust Moturakau fish record indicates a modest decrease in what are typically offshore fish taxa at that time (Figure 8). The main families involved are snapper (Lutjanidae), jacks (Carangidae), and needlefish (Belonidae), all piscivores that feed high on the food chain. A recent survey (Adams et al. 1996) indicates that Aitutaki's outer reef slopes are a prime habitat for snappers and groupers, with large stocks of both taxa currently being found there. Also at Moturakau, the herbivorous parrotfishes (Scaridae) become more common over time, along with benthic carnivores such as wrasses (Labridae), goatfish (Mullidae), and eels (Muraenidae), which occupy lower trophic levels and typify inshore areas. The archaeofaunal record also shows a reduction in Serranidae, the most abundant family overall. Serranids occupy both inshore and offshore areas and variably feed on other fish and crustaceans. In the Aitutaki case, mtDNA analysis has demonstrated that although both inshore and offshore species are represented, the most common serranid is Epinephelus merra, a small shallow-water species (Nicholls et al. 2003).

Notably, these shifts are paralleled by temporal changes in fishhook raw materials (from pearl shell or Pinctada to Turbo) and declines in fishhook abundances overall. Although hooks continue to be represented archaeologically into the early contact period, when ethnographer Peter Buck visited the island in the

\section{Moturakau Rockshelter}

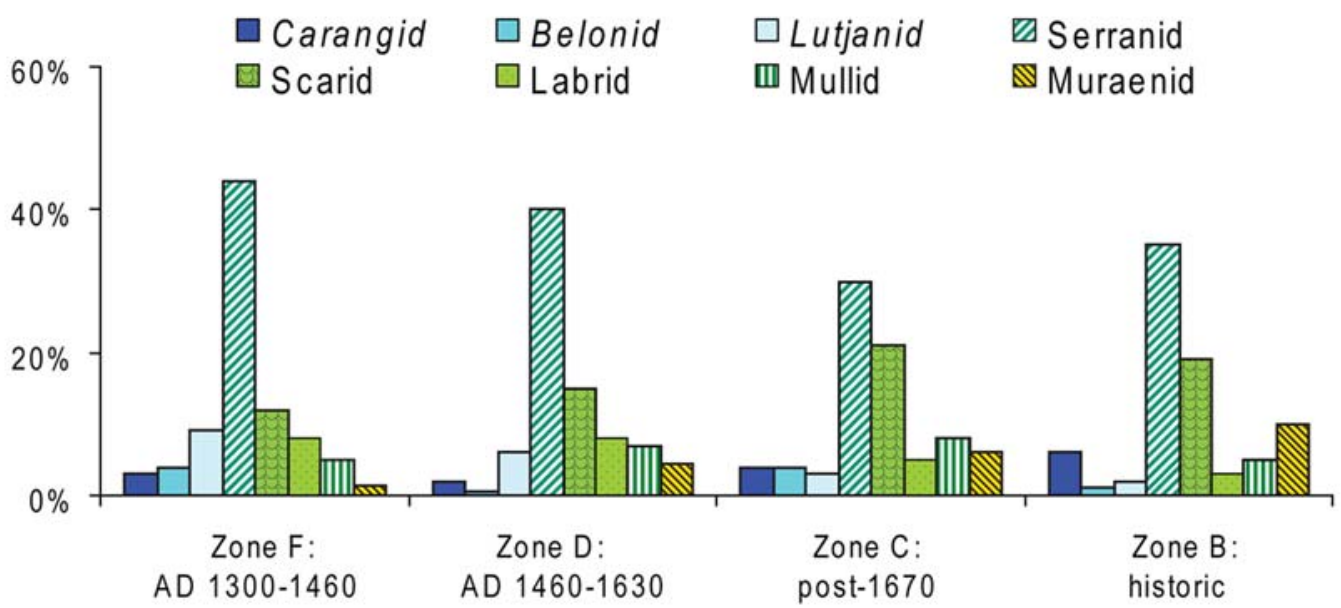

FIGURE 8. Temporal trends in major fish families; piscivores indicated by solid bars, herbivores and benthic carnivores by patterned ones (see text for details). As an aside, Westneat et al. (2005) place Scaridae taxa within the Labridae family on the basis of recent molecular results. 
early twentieth century he mainly observed mass-harvesting technologies such as nets and stone weirs. Shellfish hooks were apparently unknown and only two highly specialized hooks were recorded, the matau kio made from metal (for bonefish or Albula) and the wooden toko hook for barracuda (Sphyraena) (Buck 1927).

The stable isotope records both complement and extend these findings. They clearly demonstrate a depletion in $\delta^{15} \mathrm{~N}$ values over the three time periods outlined here (Table 10, Figure 9). The overall pattern is a loss of carnivorous fish, and those that feed on other fish (piscivores) are most likely implicated. Although there are several carnivorous fish species that frequent the lagoon, piscivorous taxa typically prefer deeper waters and commonly inhabit the outer reef slopes; this is especially true on Aitutaki, where the lagoon is relatively shallow.

The depletion in $\delta^{15} \mathrm{~N}$ values is most clearly seen in the pigs (ANOVA: $F=0.285$; $\mathrm{df}=1,13 ; P=.602)$, which are well represented in all three time periods. However, this trend is also suggested by the human data, for which there are early and late individuals. Further, the dogs, which are mainly early samples, are consistent with the pig and human findings to the degree that they also suggest elevated $\delta^{15} \mathrm{~N}$ values. It is difficult to tell when the critical changes begin because there are individuals in Period I with both high and low $\delta^{15} \mathrm{~N}$ values. Similar patterns hold in Period II, but there are fewer individuals with enriched $\delta^{15} \mathrm{~N}$ values (i.e., above $13 \%$ ) at that time. By Period III, all individuals have $\delta^{15} \mathrm{~N}$ values below $13 \%$.

Although these changes appear to postdate the fourteenth century coastal disruptions, indirectly they may be an outcome of shifting economic priorities that arose in the aftermath of successive tropical cyclones. In an earlier work, Allen (1992) argued that declines in offshore fishing stemmed from perceived or actual costs of working the more distant and risky outer reef habitat. Among the potential costs were those related to shell-hook production and construction and maintenance of seaworthy canoes. Risks included the focus on large but solitary prey, with the possibility of the "big one getting away," the dangers of capsizing outside the reef, and perhaps most important from a nutritional perspective, time away from crucial agricultural pursuits. Tropical cyclones, like those indicated in the fourteenth century, routinely batter not only local reefs but also gardens and important fruit-bearing trees like coconut and breadfruit. Climatic instability of this kind might have precipitated an economic reorientation and fostered an increased focus on the agroeconomy. The isotope evidence from Period II, indicative of

TABLE 10

Summary of Changing $\delta^{15} \mathrm{~N}$ Values across All Sites (Period III Values Shown for Comparison Only)

\begin{tabular}{|c|c|c|c|c|}
\hline Taxon & $\begin{array}{c}\text { Period I } \\
\text { Mean } \delta^{15} \mathrm{~N}\end{array}$ & $\begin{array}{c}\text { Period II } \\
\text { Mean } \delta^{15} \mathrm{~N}\end{array}$ & ANOVA & $\begin{array}{l}\text { Period III } \\
\text { Mean } \delta^{15} \mathrm{~N}\end{array}$ \\
\hline Pig & $\begin{array}{l}12.2(n=8) \\
\text { Range: } 10.1 \text { to } 15.3 \\
\text { SD } 1.8290\end{array}$ & $\begin{array}{l}11.7(n=7) \\
\text { Range: } 9.9 \text { to } 14.6 \\
\text { SD } 1.5790\end{array}$ & $\begin{array}{l}F=.285 \\
\mathrm{df}=1,13 \\
P=.602\end{array}$ & $9.8(n=3)$ \\
\hline Dog & $\begin{array}{l}11.7(n=5) \\
\text { Range: } 11.1 \text { to } 14.6 \\
\text { SD } 0.6238\end{array}$ & $\begin{array}{l}12.9(n=5) \\
\text { Range: } 11 \text { to } 14.9 \\
\text { SD } 1.6670\end{array}$ & $\begin{array}{l}F=.064 \\
\mathrm{df}=1,8 \\
P=.806\end{array}$ & $11.9(n=1)$ \\
\hline All taxa & $\begin{array}{l}12.0(n=21) \\
\text { Range: } 10.1 \text { to } 15.3 \\
\text { SD } 1.3658\end{array}$ & $\begin{array}{l}12.0(n=12) \\
\text { Range: } 9.9 \text { to } 14.9 \\
\text { SD } 1.5680\end{array}$ & $\begin{array}{l}F=.763 \\
\mathrm{df}=1,31 \\
P=.288\end{array}$ & $11.1(n=14)$ \\
\hline
\end{tabular}




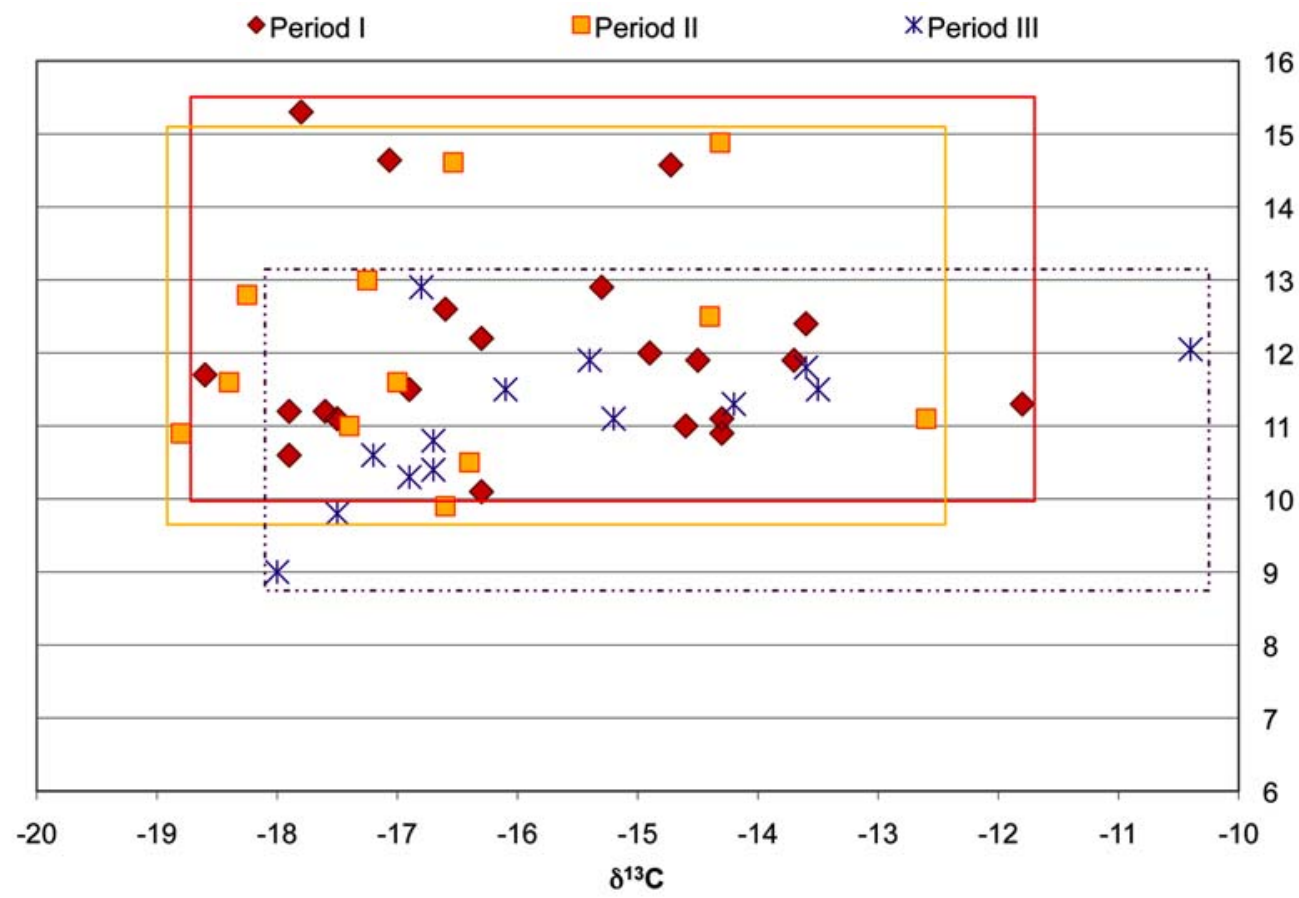

FIGURE 9. All isotope samples identified by time period; range of variability for each time period encompassed by box.

more input from terrestrial proteins and possibly a reduced reliance on marine resources, could reflect such shifting priorities.

Overall, the decrease in carnivorous fish taxa, decline in shell fishhooks, and changing $\delta^{15} \mathrm{~N}$ signatures point to important changes in how and where fish were caught. These patterns are consistent with less-frequent use of outer reef habitats where piscivorous fish congregate and where angling technologies were particularly effective. Notably, findings from modern time allocation studies (Adams et al. 1996) demonstrate that netting techniques like those described by Buck (1927) are routinely more productive than hand-line or spear fishing in terms of catch in kilograms per person-hr. Thus, inshore reef fishing might have been not only less costly and risky but also more productive. There appears to be a time lag, however, between the practices indicated by the archaeofaunal and artifact records, and the isotopic evidence for a full transition to a lower trophic level. The reasons for this lag remain unclear, and throughout all time periods there is marked variability (Figure 9). The patterns as a whole suggest a complex interplay between environmental variability (i.e., the fourteenth-century period of storminess) and cultural decisions about economic adjustments in both marine and terrestrial contexts, which ultimately ensured adequate levels of nutrition for both human populations and their domesticates.

\section{Reductions in Diet Variability}

Toward the end of prehistory, subsistence resources again may have experienced some kind of stress, possibly climatic variability or perhaps simply the effects of a growing human population on the resources of a small island. The "stress" is suggested most 


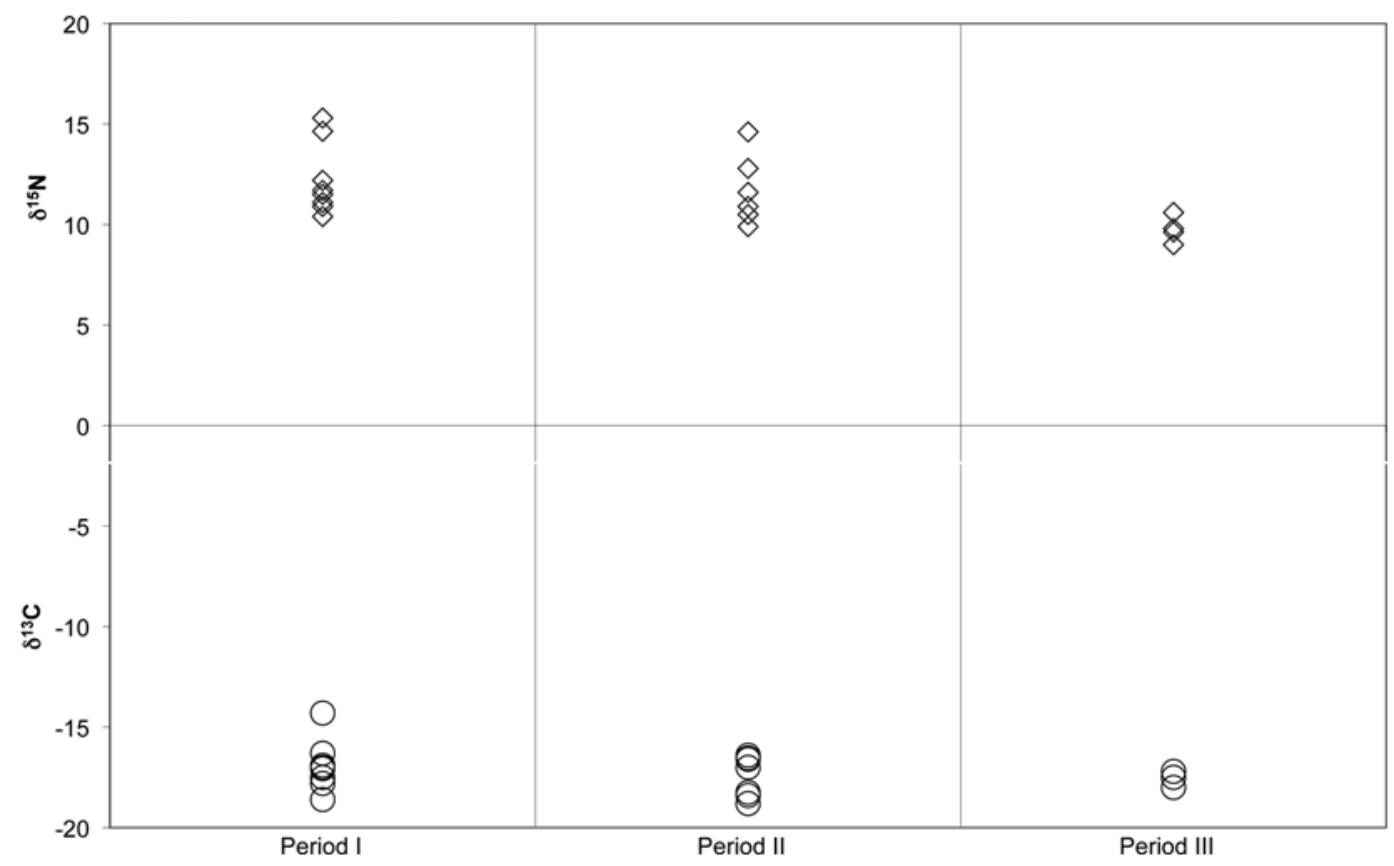

FIGURE 10. Isotopic variability in pigs over time. Circles represent individual $\delta^{13} \mathrm{C}$ values and the diamonds individual $\delta^{15} \mathrm{~N}$ values.

strongly by decreases in the isotopic variability of pig diets (Figure 10). Specifically, there is a reduction in the variability of both $\delta^{15} \mathrm{~N}$ and $\delta^{13} \mathrm{C}$ signatures after ca. A.D. 1650. This change indicates that although there were still differences in pig husbandry practices, their diet became more restricted in terms of protein sources. Increasing pressures on the available food resources of the island and a tighter control over pig diets by their owners may be indicated. However, this hypothesized shift in pig management does not explain the changes in fish bone deposition outlined earlier. The isotope values point to a significant reduction in the marine protein consumption of pigs $(F=4.58$; df $=1,16 ; P=.048)$, but that shift occurs after ca. A.D. 1650 . We cannot, however, discount the possibility that the reduced variability in pig diets is an artifact of the small size of the late sample, with only three observations, compared with the two earlier periods, where there are eight (I) and seven samples (II). Nevertheless, by European contact, reputedly both $\operatorname{dog}$ and pig had been extirpated (Williams 1837, Bligh 1937), a pattern that has been found on several other small Polynesian islands (BayPeterson 1984, Kirch 2000, Giovas 2006) and one that is often associated with interspecific competition (Oosting 1956, Begon et al. 1996).

\section{DISCUSSION}

The combined archaeofaunal and stable isotope records provide a detailed view of subsistence change on Aitutaki, one that speaks to both plant and animal food sources and is relevant not only to human but also to domesticate animal populations. Both new information and support for previously identified trends has been reviewed. Of particular interest here are the distinctive perspectives provided by these two kinds of records and 
the ways that these differences potentially relate to formation histories, taxonomic resolution, and the scale of activities (individual versus populations).

The vertebrate faunal record clearly identifies a long-term decline in fish bone deposition, one that begins in the fourteenth century and continued into the historic period. Initially, however, it was uncertain whether this trend was the result of subsistence change or some other process. At issue was the question of how the faunal assemblages had accumulated and the potential influence of nondietary processes. A number of hypotheses were considered, only some of which could be rejected.

Stable isotope records also are affected by formation processes such as the age at which a tissue develops, variability across different tissues types, certain climatic influences during life, and postdepositional diagenesis (see Ambrose and Katzenberg 2001). These issues are complex and explored in relation to the Aitutaki samples elsewhere (Craig 2009). Of particular interest here, however, is that despite quite different formation histories, both the archaeofaunal and isotopic results suggest a decline in marine resources around the fourteenth to fifteenth centuries A.D. Notably, the change is not equally well registered in the two records. The limited ability of stable isotopes to clearly differentiate between potentially important categories of Polynesian marine proteins may be an issue, with overlap in the isotopic ranges of inshore fish and other marine fauna such as shellfish and invertebrates (see values in Ambrose [1993], Valentin et al. [2006]).

Similarly, both records point to a decline in marine carnivores. However, this change is more strongly registered in the isotopic data through the depleted $\delta^{15} \mathrm{~N}$ values. Weaker expression of this trend in the archaeofaunal evidence may reflect the family level determinations that typify the fish bone study. Food preferences in some large fish families (e.g., the prominent Serranidae) are quite variable, making it difficult to categorize them with respect to diet as attempted in Figure 8 . Further, some potentially important marine carnivores have only weak archaeo- faunal signatures. Sharks, for example, were a food source in the historic period (Buck 1927) and may have been important in the past as well. They are not, however, well represented in the archaeological record relative to other fish families because most of their skeleton is cartilaginous; the same is true of rays and skates. The enriched $\delta^{15} \mathrm{~N}$ values observed in several early samples could reflect regular access to these zooarchaeologically "invisible" marine carnivores. It is interesting that the isotopic evidence indicates that the loss of enriched nitrogen sources was a gradual and long-term process, rather than an episodic event.

The two kinds of records also aid understanding of variability in the dietary experiences of species, individuals, households, and communities. In the case of the isotopic analysis, Craig (2009) has identified statistically significant differences in the human $\delta^{13} \mathrm{C}$ and $\delta^{15} \mathrm{~N}$ values that crosscut time periods and clearly reflect geography. This variability highlights the methodological problem of using single summary values, or small numbers of samples, to characterize populations even over limited geographic space, a land area of only $18.4 \mathrm{~km}^{2}$ in the Aitutaki case. Some intraspecific variability also may reflect social differences between individuals and households, variation in animal husbandry practices, and/or patterns of mobility, although samples sizes are currently too small to determine specific causes. The faunal assemblages, in turn, aid identification of larger-scale processes. The availability of archaeofaunal records from multiple locations has been particularly advantageous in identifying islandwide trends.

In comparing the isotopic results for the three species, it is worthwhile to consider whether the variability seen here is telling us something about ongoing risks and uncertainties of life on a small and isolated island. An important but perhaps not unexpected discovery is that Aitutaki's human populations occupied a central and relatively stable position in the largely anthropogenic food web at all times (Figure 5; see also Craig [2009]). The isotopic values of domesticates, in contrast, are peripheral to, and migrate around, 
those of their human managers. In this respect they may be the proverbial "canary in a coal mine," signaling the onset of prehistoric environmental and cultural perturbations. Although this idea is not entirely novel, the Aitutaki evidence provides one of the first empirical illustrations of these relationships in the Pacific context.

Recognition of differences in the amount of variability within species, however, also raises questions about how animal life span relates to isotopic patterning. Pigs, with an average life span of 2 to 3 years (the maximal age of most of the Aitutaki specimens), were isotopically the most variable. Humans, in contrast, where the values typically reflect the last two to three decades of life (Wild et al. 2000, Ubelaker et al. 2006, Hedges et al. 2007), were the most uniform. Dogs, which probably had life spans of around 5 to 7 years, were intermediate. These patterns suggest that life span could be a bias, one that affects interspecific comparisons and dietary reconstructions. Figure 11 is an attempt to model these relationships. In this example, the Year 1-2 pig consumed a diet relatively low in $\delta^{13} \mathrm{C}$, but the Year 4-6 pig had a comparatively enriched $\delta^{13} \mathrm{C}$ diet. In the human sample from the same time period (Years 1 through 6), these variations in $\delta^{13} \mathrm{C}$ are averaged; as a result, fine-grained dietary/ environmental information is potentially lost. Moreover, direct comparisons with pigs, which sample environmental variability at a different temporal scale, are not entirely appropriate. Although these life span differences add interpretive complexities, they also may be analytically useful because they potentially provide novel insights into social and environmental variability over different temporal scales. Short-lived species like pigs (i.e., quickly harvested) may tell us about multiannual variability, while longer-lived species provide decadal-scale averages (Figure 11). Exploiting these potentials, however, will require both larger and more tightly provenienced samples than are currently available.

\section{CONCLUSIONS}

The two subsistence archives reviewed here illustrate that although the most dramatic dietary changes on Polynesian islands often occurred in the immediate aftermath of colonization, human populations, nevertheless, continued to experience and respond to

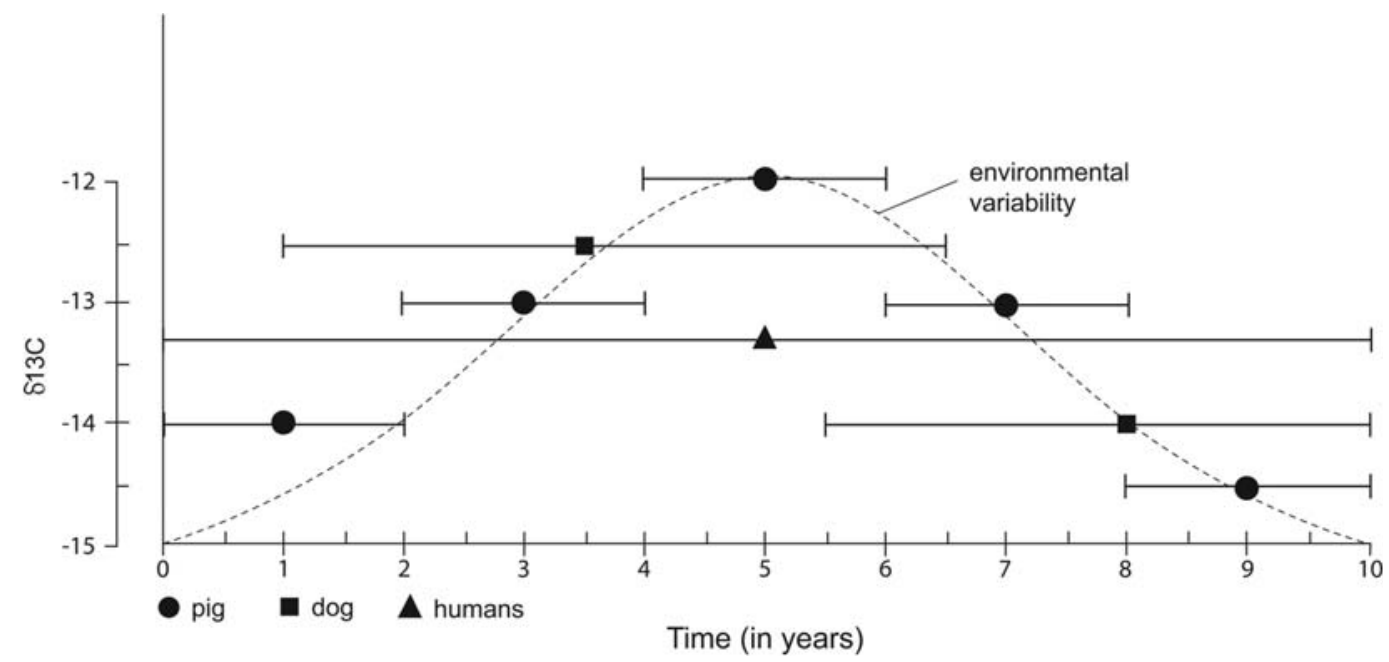

FIGURE 11. A model of how variation in animal life span might differentially record the availability of $\delta^{13} \mathrm{C}$ resources over time (assuming similar diets across the three species). Each symbol represents an individual animal, with the horizontal bars indicating their life span. The dotted line represents variation in $\delta^{13} \mathrm{C}$ over a 10 -year period. 
changing environmental and social conditions in the postcolonization period. By integrating traditional archaeofaunal assemblages, stable isotope analyses, new chronological information, and geoarchaeological records, we have identified four distinctive features of Aitutaki's subsistence economy, although, as discussed, in some cases our results are indicative rather than definitive given the small sample sizes. The key findings are (1) the established nature of Aitutaki's subsistence economy from the fourteenth century onward, (2) an islandwide decline in consumption of marine fish, (3) a decline in marine carnivore consumption, and (4) for pigs, a possible reduction in diet variability. Although broad patterns of change are apparent, the isotopic record in particular highlights variability within all three populations at all time periods, also an important finding.

Most generally, both records are consistent with the idea developed elsewhere (Allen 1992, Allen and Wallace 2007) that the currently known Aitutaki archaeological records do not represent initial settlement on this island. Throughout the sequence, agricultural food sources dominate and humans occupy a central position within this largely anthropogenic food web. The recent redating program of Allen and Wallace (2007) also identified the fourteenth century as a period of substantial coastal disruption, with two to three storm events registered in the local sedimentary records. It now appears that these events may have adversely affected local marine resources and been instrumental in long-term subsistence change. Although the evidence does not indicate a catastrophe (sensu Nunn 2000), it does hint that local reefs may have taken a couple of centuries to recover, and, by extension, marine resources may have been compromised in the period ca. A.D. 1400-1650.

By the mid- to late fifteenth century A.D., more specific changes are apparent in fishing practices; offshore fishing had began to decline, perhaps a secondary effect of processes set in motion in the fourteenth century. This change is registered in the faunal assemblages, the isotopic record, and in tool as- semblages. Most notably though, the isotopic evidence demonstrates that this was a longterm subsistence shift, and it was not until late prehistory (after ca. A.D. 1650) that depleted $\delta^{15} \mathrm{~N}$ values were uniformly recorded across the sampled individuals.

Late prehistory also may have seen increased competition between people and domesticates, particularly pigs. Reduced isotopic variability in pigs (Figure 10) may indicate that nonplant protein sources became less widely available. Pig extirpation, or at least their greatly reduced numbers, in the early contact period also speak to interspecific competition, and historic records suggest the possibility that similar processes were operating on dogs (Williams 1837, Bligh 1937).

Finally, we have also used the Aitutaki case as a platform for discussing the challenges and possibilities of integrating varied kinds of records to track and interpret dietary and subsistence change. Differences in formation processes, taxonomic resolution, and spatial and temporal scales of these records have been considered and some exciting potentials identified. The Aitutaki case illustrates how the use of multiple subsistence archives can provide textured and multiscalar understandings of subsistence change across communities and islands.

\section{ACKNOWLEDGMENTS}

Judith Littleton, Anne Katzenberg, Nancy Beaven, Scott Fitzpatrick, Michiko Intoh, and two anonymous reviewers are thanked for their invaluable advice and comments. Thanks also to Scott and Michiko for inviting our participation. Without the support of the people of Aitutaki to both of us on separate occasions, this research would not have been possible; in particular their permissions to analyze human remains are gratefully acknowledged. Peter Bellwood generously made samples from his 1978 excavations available to us. Identifications of the archaeofauna were undertaken by M.S.A., David Steadman, Lisa Nagaoka, Tim Canaday, and J.A.C., with further assistance from Virginia Butler. Graphic support was provided by Peter Quin and Mat Campbell. 


\section{Literature Cited}

Adams, T. J. H., I. Bertram, P. Dazell, M. Koroa, S. Matoto, J. Ngu, O. Terekia, and P. Tuara. 1996. Aitutaki lagoon fishery. http://www.spc.int/coastfish/reports/ ICFMAP/cooks/aitfinal.htm

Allen, M. S. 1992. Dynamic landscapes and human subsistence: Archaeological investigations on Aitutaki Island, southern Cook Islands. Ph.D. diss., University of Washington. University Microfilms International, Ann Arbor, Michigan.

- 1994. The chronology of coastal morphogenesis and human settlement on Aitutaki, southern Cook Islands, Polynesia. Radiocarbon 36:59-71. 1998. Holocene sea-level change on Aitutaki, Cook Islands: Landscape change and human response. J. Coastal Res.14:1022.

. 2002. Resolving long-term change in Polynesian marine fisheries. Asian Perspect. 41:195-212.

Allen, M. S., and R. Wallace. 2007. New evidence from the East Polynesian gateway: Substantive and methodological results from Aitutaki, southern Cook Islands. Radiocarbon 49:1163-1179.

Ambrose, S. H. 1990. Preparation and characterization of bone and tooth collagen for isotopic analysis. J. Archaeol. Sci. 17:431-451.

1993. Isotopic analysis of palaeodiets: Methodological and interpretive considerations. Pages 59-130 in M. K. Sandford, ed. Investigations of ancient human tissue: Chemical analyses in anthropology. Gordon and Breach Science Publishers, Amsterdam.

Ambrose, S. H., B. M. Butler, D. B. Hanson, R. L. Hunter-Anderson, and H. W. Krueger. 1997. Stable isotopic analysis of human diet in the Marianas Archipelago, western Pacific. Am. J. Phys. Anthropol. 104:343-361.

Ambrose, S. H., and M. A. Katzenberg, eds. 2001. Biogeo-chemical approaches to paleodietary analysis in archaeology. Advances in archaeological and museum science. Plenum Press, New York.
Ambrose, S. H., and J. Krigbaum. 2003. Bone chemistry and bioarchaeology. J. Anthropol. Archeol. 22 (3): 193-199.

Ambrose, S. H., and L. Norr. 1993. Experimental evidence for the relationship of the carbon isotope rations of whole diet and dietary protein to those of bone collagen and carbonate. Pages 271-238 in J. B. Lambert and G. Grupe, eds. Prehistoric human bone: Archaeology at the molecular level. Springer-Verlag, Berlin.

Anderson, A. 1994. Palaeoenvironmental evidence of island colonization: A response. Antiquity 68:845-847.

. 1997. Prehistoric Polynesian impact on the New Zealand environment: Te whenua hou. Pages 271-283 in P. V. Kirch and T. L. Hunt, eds. Historical ecology in the Pacific islands. Yale University Press, New Haven, Connecticut.

- 2001. No meat on that beautiful shore: The prehistoric abandonment of Polynesian islands. Int. J. Osteoarchaeol. 11:14-23.

Barber, I. G. 2003. Sea, land, and fish: Spatial relationships and the archaeology of South Island Maori fishing. World Archaeol. 35:434-448.

Bay-Peterson, J. 1984. Competition for resources: The role of pig and dog in the Polynesian agricultural economy. J. Soc. Océanistes 77:121-129.

Beavan-Athfield, N., R. C. Green, J. A. Craig, B. McFadgen, and S. Bickler. 2008. Influence of marine sources on ${ }^{14} \mathrm{C}$ ages: Isotopic data from Watom Island, Papua New Guinea, inhumations and pig teeth in light of new dietary standards. J. R. Soc. N. Z. 38:1-23.

Begon, M., J. L. Harper, and C. R. Townsend. 1996. Ecology: Individuals, populations, and communities. 3rd ed. Blackwell Science, Oxford.

Bellwood, P. 1978. Archaeological research in the Cook Islands. Pacific Anthropological Records 27. B. P. Bishop Museum, Honolulu.

Bligh, W. 1937. The log of the Bounty. Vol. 2. Golden Cockerel Press. London.

Bösl, C., G. Grupe, and J. Peters. 2006. A late Neolithic vertebrate food web based on 
stable isotope analyses. Int. J. Osteoarchaeol. 16:296-315.

Bronk Ramsey, C. 1995. Radiocarbon calibration and analysis of stratigraphy: The OxCal program. Radiocarbon 37:425-430.

. 2001. Development of the radiocarbon program OxCal. Radiocarbon 43 (2A): 355-363.

Buck, P. (Te Rangi Hiroa). 1927. The material culture of the Cook Islands (Aitutaki). Memoirs of the Board of Maori Ethnological Research, New Plymouth, New Zealand.

Butler, V. L. 2001. Changing fish use on Mangaia, southern Cook Islands: Resource depression and the prey choice model. Int. J. Osteoarchaeol. 11:88-100.

Charnov, E. L., G. H. Orians, and K. Hyatt. 1976. Ecological implications of resource depression. Am. Nat. 110:247-259.

Colman, L. J. 1998. Rattus exulans as a food source: The case of Aitutaki. M. A. thesis, University of Auckland. Auckland, New Zealand.

Coltrain, J. B., J. M. Harris, T. E. Cerling, J. R. Ehleringer, M. D. Dearing, J. Ward, and J. Allen. 2004. Rancho La Brea stable isotope biogeochemistry and its implications for the palaeoecology of late Pleistocene coastal southern California. Palaeogeogr., Palaeoclimatol., Palaeoecol. 205:199-219.

Craig, J. A. 2009. Stable isotope analysis of prehistoric human and commensal diet on Aitutaki, southern Cook Islands. Ph.D. diss., University of Auckland, Auckland, New Zealand.

DeNiro, M. J. 1985. Postmortem preservation and alteration of in vivo bone collagen isotope ratios in relation to palaeodietary reconstruction. Nature (Lond.) 317:806809.

. 1987. Stable isotopy and archaeology. Am. Sci. 75:182-191.

de Scally, F. 2008. Historical tropical cyclone activity and impacts in the Cook Islands. Pac. Sci. 62:443-459.

Dickinson, W. R. 2003. Impact of midHolocene hydro-isostatic highstand in regional sea level on habitability of islands in Pacific Oceania.J. Coastal Res. 19:489-502.
Fitzpatrick, S. M., and O. Kataoka. 2005. Prehistoric fishing in Palau, Micronesia: Evidence from the northern Rock Islands. Archaeol. Oceania 40:1-13.

Fogel, M. L., N. Tuross, B. Johnson, and G. H. Miller. 1997. Biogeochemical record of ancient humans. Org. Geochem. 27:275-287.

Fosberg, F. R. 1975. Vascular plants of Aitutaki. Pages 74-84 in D. R. Stoddart and P. E. Gibbs, eds. Almost-atoll of Aitutaki: Reef studies in the Cook Islands, South Pacific. Atoll Res. Bull. 190.

Giovas, C. 2006. No pig atoll: Island biogeography and the extirpation of a Polynesian domesticate. Asian Perspect. 45: 69-95.

Grayson, D. K. 1984. Quantitative zooarchaeology: Topics in the analysis of archaeological faunas. Academic Press, New York.

Guralnick, L. J., G. Edwards, M. S. B. Ku, B. Hockema, and V. R. Franceschi. 2002. Photosynthetic and anatomical characteristics in the C-4-crassulacean acid metabolism-cycling plant, Portulaca grandiflora. Funct. Plant Biol. 29:763-773.

Hedges, R. E. M., J. G. Clement, C. D. L. Thomas, and T. C. O'Connell. 2007. Collagen turnover in the adult femoral midshaft: Modeled from anthropogenic radiocarbon tracer measurements. Am. J. Phys. Anthropol. 122:808-816.

Herring, D. A., S. R. Saunders, and M. A. Katzenberg. 1998. Investigating the weaning process in past populations. Am. J. Phys. Anthropol. 105:425-439.

Katzenberg, M. A. 2008. Stable isotope analysis: A tool for studying past diet, demography, and life history. Pages 413-440 in M. A. Katzenberg and S. R. Saunders, eds. Biological anthropology of the human skeleton. 2nd ed. Wiley-Liss, Hoboken, New Jersey.

Katzenberg, M. A., H. P. Schwarcz, M. Knyf, and F. J. Melbye. 1995. Stable isotope evidence for maize horticulture and paleodiet in southern Ontario, Canada. Am. Antiquity 60:335-350.

Kirch, P. V. 1973. Prehistoric subsistence patterns in the northern Marquesas Is- 
lands, French Polynesia. Archaeol. Phys. Anthropol. Oceania 8:24-40. 2000. Pigs, humans and trophic competition on small Oceanic islands. Pages 427-439 in A. Anderson and T. Murray, eds. Australian archaeologist: Collected papers in honour of Jim Allen. Coombs Academic Publishing, Canberra.

Kirch, P. V., and J. Ellison. 1994. Paleoenvironmental evidence for human colonization of remote Oceanic islands. Antiquity 68:310-321.

Kirch, P. V., D. W. Steadman, V. L. Butler, J. Hather, and M. I. Weisler. 1995. Prehistory and human ecology in eastern Polynesia: Excavations at Tangatatau Rockshelter, Mangaia. Archaeol. Oceania 30:47-65.

Kirch, P. V., and D. E. Yen. 1982. Tikopia: The prehistory and ecology of a Polynesian outlier. Bernice P. Bishop Mus. Bull. 238.

Larsen, C. S. 1997. Bioarchaeology: Interpreting behaviour from the human skeleton. Cambridge University Press, Cambridge.

Leach, B. F., J. Davidson, K. Fraser, and A. Anderson. 1999. Pre-European catches of barracouta, Thyristes atun, at Long Beach and Shag River Mouth, Otago, New Zealand. Archaeofauna 8:11-30.

Leach, B. F., C. J. Quinn, and G. L. Lyon. 1996. A stochastic approach to the reconstruction of prehistoric human diet in the Pacific region from bone isotope signatures. Tuhinga Records Museum of N. Z., Te Papa Tongarewa 8:1-54.

Leach, B. F., C. J. Quinn, G. L. Lyon, A. Haystead, and D. B. Myers. 1998. Evidence of Prehistoric Lapita diet at Watom Island, Papua New Guinea, using stable isotopes. N. Z. J. Archaeol. 20:149-159.

Leach, B. F., C. J. Quinn, J. Morrison, and G. Lyon. 2003. The use of multiple isotope signatures in reconstructing prehistoric human diet from archaeological bone from Pacific and New Zealand. N. Z. J. Archaeol. 23:31-98.

Lee-Thorp, J. A. 2008. On isotopes and old bones. Archaeometry 50 (6): 925-950.
Masse, W. B., J. Liston, J. Carucci, and J. S. Athens. 2006. Evaluating the effects of climate change on environment, resource depletion, and culture in the Palau Islands between A.D. 1200 and 1600. Quat. Int. 151:106-132.

McAlister, A. J. 2002. Prehistoric fishing at Fakaofo, Tokelau: A case for resource depression on a small atoll. M. A. thesis, University of Auckland, Auckland, New Zealand.

McCormac, F. G., A. G. Hogg, P. G. Blackwell, C. E. Buck, T. F. G. Higham, and P. J. Reimer. 2004. SHCal04 Southern Hemisphere Calibration 0-1000 cal BP. Radiocarbon 46:1087-1092.

Minagawa, M., and E. Wada. 1984. Stepwise enrichment of $\delta^{15} \mathrm{~N}$ along food chains: Further evidence and the relations between $\delta^{15} \mathrm{~N}$ and animal age. Geochim. Cosmochim. Acta 48:1135-1140.

Morrison, A. E., and E. E. Cochrane. 2008. Investigating shellfish deposition and landscape history at the Natia Beach Site, Fiji. J. Archaeol. Sci. 35:2387-2399.

Morrison, A. E., and T. L. Hunt. 2007. Human impacts on the nearshore environment: An archaeological case study from Kaua'i, Hawaiian Islands. Pac. Sci. 61:325345.

Nagaoka, L. 2002a. Explaining subsistence change in southern New Zealand using foraging theory models. World Archaeol. $34: 84-102$

$2002 b$. The effects of resource depression on foraging efficiency, diet breadth and patch choice use in southern New Zealand. J. Anthropol. Archaeol. 21:419-442.

Nicholls, A., E. Matisoo-Smith, and M. S. Allen. 2003. A novel application of molecular techniques to Pacific achaeofish remains. Archaeometry 45:133-147.

Nunn, P. D. 2000. Environmental catastrophe in the Pacific islands around A.D. 1300. Geoarchaeology 15:715-740.

Oosting, H. J. 1956. The study of plant communities: An introduction to plant ecology. W. H. Freeman and Co., San Francisco. 
Pate, F. D., J. L. Craib, and G. M. Heathcote. 2001. Stable isotopic analysis of prehistoric human diet in the Mariana Islands, western Pacific. Aust. Archaeol. 52:1-4.

Pirazzoli, P., and L. Montaggioni. 1986. Late Holocene sea-level changes in the Northwest Tuamotu Islands, French Polynesia. Quat. Res. 25:350-368.

Rolett, B. V. 1998. Hanamiai: Prehistoric colonization and cultural change in the Marquesas Islands (East Polynesia). Yale University Publications in Anthropology 81, New Haven, Connecticut.

Salinger, M. J., and P. Lefale. 2005. The occurrence and predictability of extreme events over the Southwest Pacific with particular reference to ENSO. Pages 3939 in R. P. Motha, M. V. K. Siva Kumar, and H. P. Das, eds. Natural disasters and extreme events in agriculture: Impacts and mitigation. Springer-Verlag, Heidelberg.

Schoeninger, M. J. 1989. Reconstructing prehistoric human diet. Pages 38-67 in T. D. Price, ed. The chemistry of prehistoric human bone. Cambridge University Press, Cambridge.

Schoeninger, M. J., and M. J. DeNiro. 1984. Nitrogen and carbon isotopic composition of bone collagen from marine and terrestrial animals. Geochim. Cosmochim. Acta 48:625-639.

Schoeninger, M. J., M. J. DeNiro, and H. P. Tauber. 1983. Stable nitrogen isotope ratios of bone collagen reflect marine and terrestrial components of prehistoric human diet. Science (Washington, D.C.) 220:1381-1383.

Spennemann, D. H. R. 1987. Availability of shellfish resources on prehistoric Tongatapu, Tonga: Effects of human predation and changing environment. Archaeol. Oceania 22:81-96.

Steadman, D. W. 1991. Extinct and extirpated birds from Aitutaki and Atiu, southern Cook Islands. Pac. Sci. 45:325-347.

. 2006. Extinction and biogeography of tropical Pacific birds. University of Chicago Press, Chicago.

Steadman, D. W., C. Vargas, and F. Cristino. 1994. Stratigraphy, chronology, and cul- tural context of an early faunal assemblage from Easter Island. Asian Perspect. 33:7996.

Stoddart, D. R. 1985. Hurricane effects on coral reefs: Conclusion. Proc. 5th Int. Coral Reef Symp. 3:349-350.

Stoddart, D. R., and P. E. Gibbs, eds. 1975. Almost-atoll of Aitutaki: Reef studies in the Cook Islands, South Pacific. Atoll Res. Bull. 190.

Tauber, H. P. $1981 .{ }^{13} \mathrm{C}$ evidence for dietary habits of prehistoric man in Denmark. Nature (Lond.) 292:332-333.

Ubelaker, D. H., B. A. Buchholz, and J. E. B. Stewart. 2006. Analysis of artificial radiocarbon in different skeletal and dental tissue types to evaluate date of death. J. Forensic Sci. 51 (3): 484-488.

Valentin, F., H. Bocherens, B. Gratuze, and C. Sand. 2006. Dietary patterns during the late prehistoric/historic period in $\mathrm{Ci}$ kobia island (Fiji): Insights from stable isotopes and dental pathologies. J. Archaeol. Sci. 3:1396-1410.

Vogel, J. C., and N. M. van der Merwe. 1977. Isotopic evidence for early maize cultivation in New York State. Am. Antiquity 42:238-242.

Walter, R. 1998. Anai'o: The archaeology of a fourteenth century Polynesian community in the Cook Islands. N. Z. Archaeol. Association Monograph 22. Auckland.

Weisler, M. I. 1995. Henderson Island prehistory: Colonization and extinction on a remote Polynesian island. Biol. J. Linn. Soc. 56:377-404.

Westneat, M. W., M. E. Alfaro, P. C. Wainwright, D. R. Bellwood, J. R. Grubich, J. L. Fessler, K. D. Clements, and L. L. Smith. 2005. Local phylogenetic divergence and global evolutionary convergence of skull function in reef fishes of the family Labridae. Proc. R. Soc. Lond. B 272:993-1000.

Whistler, W. A. 1991. Polynesian plant introductions. Pages 41-66 in P. A. Cox and S. A. Banack, eds. Islands, plants and Polynesians: An introduction to Polynesian ethnobotany. Dioscorides Press, Portland, Oregon. 
Wild, E. M., K. A. Arlamovsky, R. Golser, W. Kutschera, A. Priller, S. Puchegger, W. Rom, P. Steier, and W. Vycudilik. 2000. ${ }^{14} \mathrm{C}$ dating with the bomb peak: An application to forensic medicine. Nucl. Instr. Meth. Phys. Res. B 172:944-950.
Williams, J. 1837. A narrative of missionary enterprises in the South Sea islands: With remarks upon the natural history of the islands, origin, languages, traditions, and usages of the inhabitants. J. Snow, London. 